\title{
A low-cost pipeline for soil microbiome profiling
}

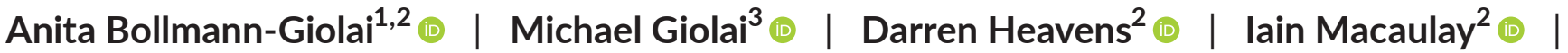 \\ Jacob Malone $e^{1,4}$ @ | Matthew D. Clark ${ }^{3,4}$ @
}

\footnotetext{
${ }^{1}$ John Innes Centre (JIC, Norwich, UK

${ }^{2}$ Earlham Institute (EI, Norwich, UK

${ }^{3}$ Natural History Museum (NHM, London, UK

${ }^{4}$ University of East Anglia, Norwich, UK
}

\section{Correspondence}

Jacob Malone, John Innes Centre (JIC), Norwich Research Park, Norwich NR4

7UH, UK.

Email: jacob.malone@jic.ac.uk

Matthew D. Clark, Natural History Museum (NHM), London SW7 5BD, UK.

Email: matt.clark@nhm.ac.uk

Funding information

Biotechnology and Biological Sciences Research Council, Grant/Award Number: BBS/E/J/000PR9797; John Innes

Foundation; Natural History Museum

\begin{abstract}
Common bottlenecks in environmental and crop microbiome studies are the consumable and personnel costs necessary for genomic DNA extraction and sequencing library construction. This is harder for challenging environmental samples such as soil, which is rich in Polymerase Chain Reaction (PCR) inhibitors. To address this, we have established a low-cost genomic DNA extraction method for soil samples. We also present an Illumina-compatible $16 \mathrm{~S}$ and ITS rRNA gene amplicon library preparation workflow that uses common laboratory equipment. We evaluated the performance of our genomic DNA extraction method against two leading commercial soil genomic DNA kits (MoBio PowerSoil ${ }^{\circledR}$ and MP Biomedicals ${ }^{\text {TM }}$ FastDNA ${ }^{\text {TM }}$ SPIN) and a recently published non-commercial extraction method by Zou et al. (PLoS Biology, 15, e2003916, 2017). Our benchmarking experiment used four different soil types (coniferous, broad-leafed, and mixed forest plus a standardized cereal crop compost mix) assessing the quality and quantity of the extracted genomic DNA by analyzing sequence variants of $16 \mathrm{~S} \mathrm{V4}$ and ITS rRNA amplicons. We found that our genomic DNA extraction method compares well to both commercially available genomic DNA

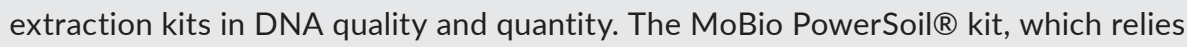
on silica column-based DNA extraction with extensive washing, delivered the cleanest genomic DNA, for example, best A260:A280 and A260:A230 absorbance ratios. The MP Biomedicals ${ }^{\mathrm{TM}}$ FastDNA ${ }^{\mathrm{TM}}$ SPIN kit, which uses a large amount of binding material, yielded the most genomic DNA. Our method fits between the two commercial kits, producing both good yields and clean genomic DNA with fragment sizes of approximately $10 \mathrm{~kb}$. Comparative analysis of detected amplicon sequence variants shows that our method correlates well with the two commercial kits. Here, we present a low-cost genomic DNA extraction method for soil samples that can be coupled to an Illumina-compatible simple two-step amplicon library construction workflow for 16S V4 and ITS marker genes. Our method delivers high-quality genomic DNA at a fraction of the cost of commercial kits and enables cost-effective, large-scale amplicon sequencing projects. Notably, our extracted gDNA molecules are long enough to be suitable for downstream techniques such as full gene sequencing or
\end{abstract}

This is an open access article under the terms of the Creative Commons Attribution License, which permits use, distribution and reproduction in any medium, 
even metagenomics shotgun approaches using long reads (PacBio or Nanopore), 10x Genomics linked reads, and Dovetail genomics.

KEYWORDS

amplicon library construction, DNA cleanup, gDNA extraction, microbiome, soil

\section{1 | INTRODUCTION}

In the last decade, microbiome studies have been increasing rapidly in popularity, from 4505 publications by December 2010 to 66,250 publications by February 2020 (PubMed reports for search term "microbiome"). Next-generation sequencing (NGS) has made microbiome studies more accessible to a wider audience of researchers through increases in throughput and falling costs (Schwarze et al., 2020). Common remaining bottlenecks for larger-scale environmental microbiome studies are the price and the hands-on time required for NGS-quality genomic DNA (gDNA) extraction and NGS library preparation. Studies sampling inhibitor-rich materials such as soil (Bahram et al., 2018; Walters et al., 2018) are further restricted to the use of specialist commercial kits (costing up to $\$ 10.26$ per extraction; Marotz et al., 2017). This cost, coupled with the many handling steps, can limit studies to smaller sample numbers. Custom gDNA extraction workflows have been described, but many current methods are low in extraction yield, throughput and often not tested for NGS or microbiome purposes or optimized for soil (Abdel-Latif \& Osman, 2017; Zou et al., 2017). Commonly used protocols for nucleic acid purification are often column and centrifuge based, which are more laborious, harder to automate and so not easily used in a high-throughput manner or scaled economically (Hamedi et al., 2016; Miao et al., 2014; Narayan et al., 2016; Oberacker et al., 2019). More recent open-source protocols for rapid DNA purification using coated magnetic particles (Oberacker et al., 2019) or even cellulose (Zou et al., 2017) address many of these shortcomings. Carboxylated or silica-coated magnetic particles as described by Oberacker et al. (2019) are commonly used in most NGS laboratories and can be readily automated, thus driving down costs effectively (Fisher et al., 2011). Researchers face a similar bottleneck with Illumina amplicon library constructions for microbiome typing (e.g., using 16S, ITS or $18 \mathrm{~S}$ markers) as for nucleic acid extraction. Commercial kits are limited to a small number of barcoded libraries (Minich, Humphrey, et al., 2018), while specialist workflows (e.g., the Earth Microbiome Project benchmarked protocols) use custom sequencing primers and therefore cannot be processed using standard Illumina protocols. This limits the choice of the available sequencing provider and affects throughput and sequencing prices (Walters et al., 2016) restricting many projects to lower throughput in-house platforms such as the Illumina MiSeq. For comparison, the Illumina MiSeq v2 500cycle kit has an $8.5 \mathrm{~Gb}$ maximal output whereas the NovaSeq 6000 SP 500-cycle kit has a 400 Gb maximal output (Bahram et al., 2018; Bartoli et al., 2008; Thiergart et al., 2020).
In scenarios with a high number of low biomass and inhibitor-rich samples such as rhizosphere and soil (Lakay et al., 2007; Zhou et al., 1996), it is therefore often too costly to perform large-scale amplicon sequencing projects with a sufficient number of samples necessary for robust statistical analysis (Bahram et al., 2018; Kelly et al., 2015). To address this, we implemented a soil DNA extraction workflow (hereafter referred to as the SDE method) by combining aluminum sulfate based humic acid removal with a magnetic bead gDNA cleanup (Figure 1) and a two-step PCR protocol creating Illumina sequencing-ready libraries. We show equal or better gDNA extraction performance from various soil types in comparison with two commercial kits and a recently published non-commercial extraction method by Zou et al. (2017; Figure A1). Further, we achieve this at a fraction of the cost per extraction (SDE: \$0.36, MP Biomedicals ${ }^{\text {TM }}$ FastDNA $^{\text {TM }}$ SPIN: \$10.26, MoBio (now QIAGEN) PowerSoil $($ : \$5.75). For our dual-indexed two-step Illuminacompatible amplicon library preparation protocol, we calculated a total number of 2304 dual-barcoded sequencing libraries with a minimal Hamming distance of 4 and a cost of \$2.5 per library preparation. With 438 custom-designed barcodes on each end, our protocol in principle could be easily expanded to 191,844 samples per lane, sufficient for NovaSeq scale (e.g., 10 billion reads at 50,000 reads per sample). The combination of our two new protocols therefore allows better utilization of state-of-the-art Illumina sequencing platforms to perform large-scale amplicon-based microbiome studies at a reduced cost.

\section{2 | MATERIALS AND METHODS}

\subsection{Soil material collection}

We selected three different woodlands, as this captured different soil characteristics (Augusto et al., 2002; Zhou et al., 1996): a coniferous forest (52.661750, 1.095444, UK), a mixed forest (46.394474, 11.235371, Italy), and a broad-leafed forest (46.454682, 11.301284, Italy). We sampled the soil material from the topsoil after removing the litter layer into sterile $50 \mathrm{ml}$ conical tubes (Supplier Starlab (UK) Ltd, E1450-0800) using nitrile gloves and a sterilized shovel. The sampled material was stored in a mobile refrigerator during transportation to the laboratory where the soil was stored at $4{ }^{\circ} \mathrm{C}$ until used for gDNA extraction. The cereal compost mix was collected in the same manner but obtained from the John Innes Horticulture facilities (Norwich, UK). 

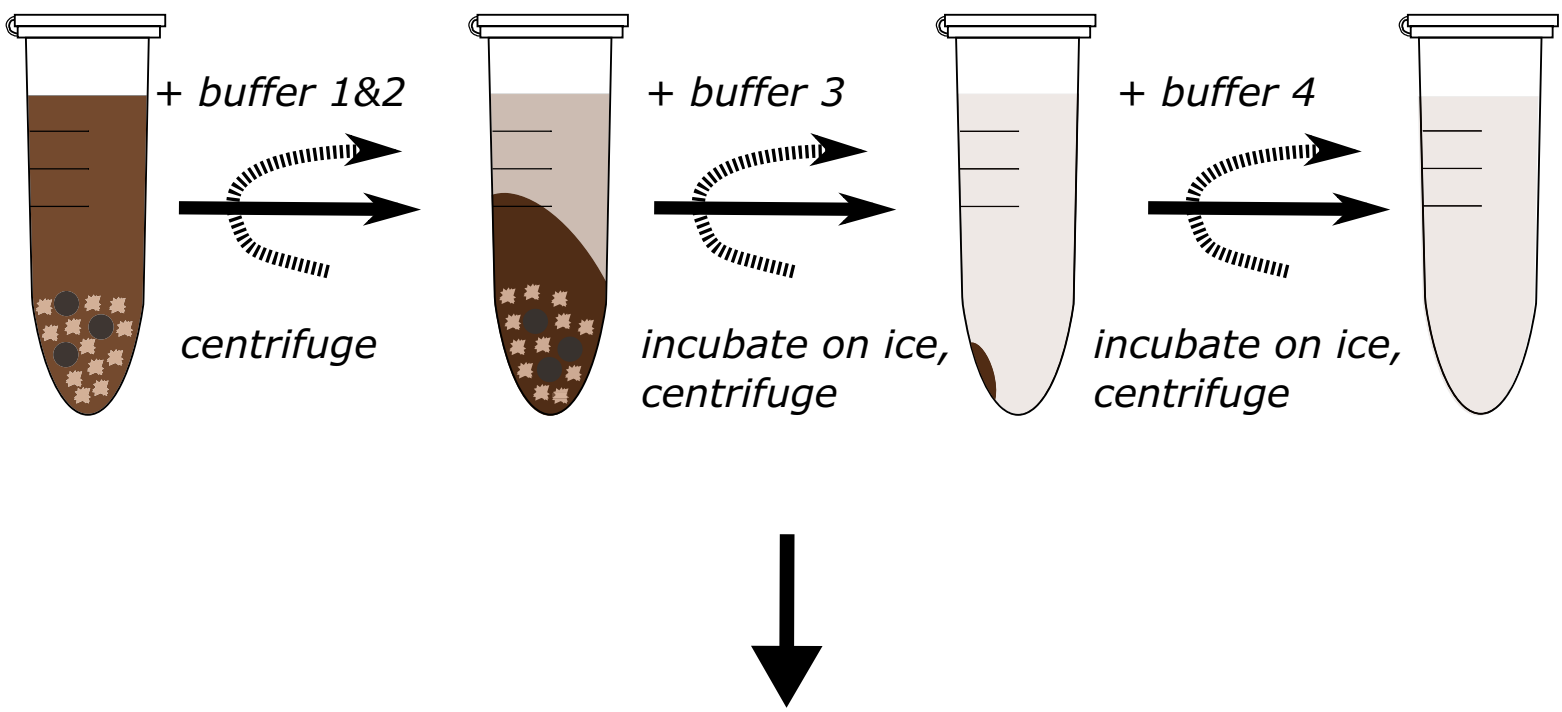

\section{magnetic bead based gDNA clean-up: in tubes or 96-well plates}

gDNA binding

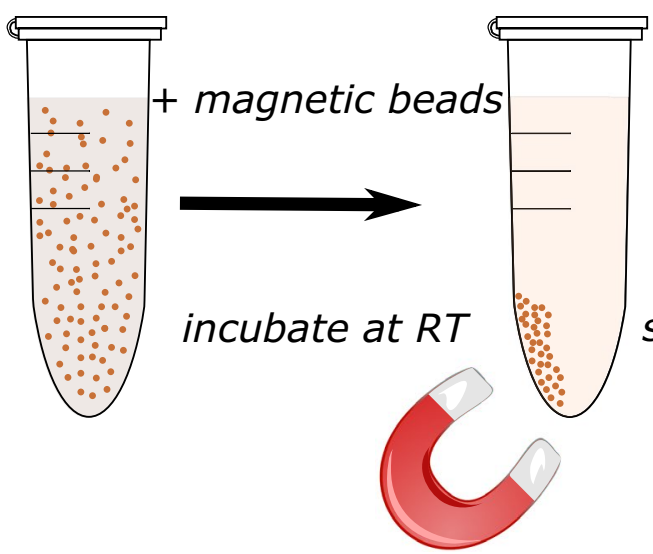

washing

gDNA release

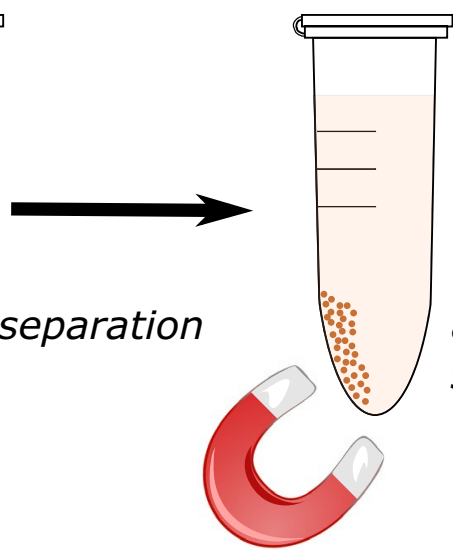

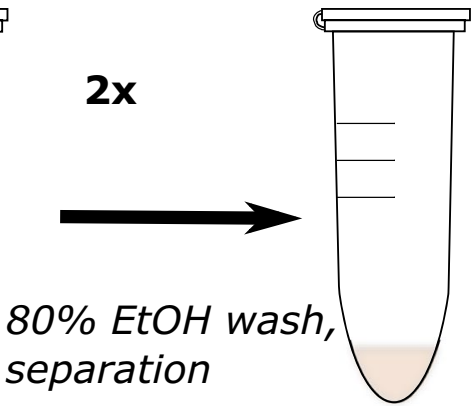

FIGURE 1 Overview of the SDE soil gDNA extraction protocol: The genomic DNA extraction protocol is divided into two parts, the gDNA extraction and the gDNA cleanup. The soil material is ground with buffer one and two, following centrifugation the proteins are removed with buffer three and incubation on ice. After centrifugation, humic acid is removed by using buffer four and incubation on ice. The extracted genomic DNA is then ready for cleanup after centrifugation. The gDNA cleanup is performed using magnetic bead-based isopropanol precipitation of the gDNA, plus two bead washing steps with $80 \%$ Ethanol. The gDNA may then be eluted into a buffer of choice-in our case TE. gDNA, genomic DNA; SDE, soil gDNA extraction method

\section{2 | MoBio PowerSoil $囚$ DNA Isolation Kit}

The kit was applied following the manufacturer's instructions with one alteration. The soil material was ground using the Geno/Grinder
(SPEX SamplePrep 2010) for $1 \mathrm{~min}$ at $1750 \mathrm{rpm}$ using the supplied grinding stones. The active hands-on time without incubation and centrifugation times is $16 \mathrm{~min}$ per extraction (S1, https://doi. org/10.5281/zenodo.4060156). 


\section{3 | MP Biomedicals ${ }^{\mathrm{TM}}$ FastDNA $^{\mathrm{TM}}$ SPIN Kit for Soil}

The kit was applied following the manufacturer's instructions with no alterations, using the recommended Fastprep machine (Fastprep24, MP BIO) for the grinding step. The active hands-on time without incubation and centrifugation times is 10 min per extraction (S1, https://doi.org/10.5281/zenodo.4060156).

\section{4 | Paperdisk method}

The paperdisk extraction used is based on the protocol by Zou et al. (2017). We followed the protocol as described previously (Zou et al., 2017) with one alteration: instead of using one paperdisk (Whatman qualitative filter paper, WHA1001070; Sigma-Aldrich Co Ltd.) per extraction, in an attempt to perform three technical PCR replicates on the same extraction, we added four disks to the extraction buffer. We washed each disk using $200 \mu \mathrm{l}$ wash buffer per disk. To determine the extracted concentration of DNA, we transferred one disk to $50 \mu \mathrm{l}$ TE buffer (10 mM Tris- $\mathrm{HCl}$ pH 8.0, 1 mM EDTA) for $4 \mathrm{hr}$ and measured the gDNA concentration using a Qubit 2.0 Fluorometer (Thermo Fisher Scientific) with Qubit dsDNA BR DNA assay kit reagents (Q32853; Thermo Fisher Scientific). The remaining three disks were used in the PCR reactions with one disk per reaction, as described by Zou et al. (2017).

\subsection{SDE method}

A total of $250 \mathrm{mg}$ soil was transferred to a $2 \mathrm{ml}$ tube containing $300 \mu$ s sterile $1 \mathrm{~mm}$ diameter garnet particles (Stratech Scientific Ltd, 11079110gar-BSP, Biospec products) and three metal $4 \mathrm{~mm}$ bearings (grade 1000 hardened 1010 Carbon steel ball bearings, Simply Bearings). Before grinding, we added $750 \mu$ Buffer 1 (181 mM Trisodium phosphate, $121 \mathrm{mM}$ guanidinium thiocyanate, $0.22 \mu \mathrm{M}$ sterile filtered with Sartorius UK Ltd, 16532K) and $60 \mu$ l Buffer 2 (150 mM NaCl, 4\% SDS, 0.5 M Tris pH7, $0.22 \mu \mathrm{M}$ sterile filtered) to each tube. To lyse the bacterial and fungal cells, the tubes were transferred to the Genogrinder (Spex SamplePrep, 2010) and run for $1 \mathrm{~min}$ at $1750 \mathrm{rpm}$. We centrifuged the tubes at 17,000 $\mathrm{g}$ for $2 \mathrm{~min}$ to pellet debris, and $450 \mu \mathrm{l}$ supernatant was transferred to a new $2 \mathrm{ml}$ tube, mixed with $250 \mu \mathrm{l}$ Buffer 3 (133 mM Ammonium acetate, $0.22 \mu \mathrm{M}$ sterile filtered), and incubated for $10 \mathrm{~min}$ on ice to precipitate proteins and SDS. We centrifuged the tubes at 17,000 rcf for $3 \mathrm{~min}$ and transferred $500 \mu \mathrm{l}$ clear supernatant to a new $2 \mathrm{ml}$ tube. The supernatant was mixed with $200 \mu \mathrm{l}$ Buffer $4(60 \mathrm{mM}$ aluminum sulfate, $0.22 \mu \mathrm{M}$ sterile filtered), and the reaction incubated for $10 \mathrm{~min}$ on ice to enable additional protein precipitation and humic acid removal. Aluminum sulfate has previously been shown to be effective for humic acid (an enzymatic inhibitor) removal of soil material (Dong et al., 2006). We centrifuged the reaction at $17,000 \mathrm{rcf}$ for $10 \mathrm{~min}$ and transferred either $600 \mu \mathrm{l}$ supernatant to a $1.5 \mathrm{ml}$ tube or $140 \mu \mathrm{l}$ supernatant to a 96 -well plate. Supernatants were stored at $-20^{\circ} \mathrm{C}$ until further use. Buffer composition for Buffer 1, 2, 3, and 4 adapted from Brolaski et al. (2008).

\subsection{SDE single tube gDNA cleanup}

To perform gDNA cleanups in single tubes, we prepared $10 \mu \mathrm{l}$ magnetic beads (Sera-Mag Carboxylate-Modified Magnetic Particles (Hydrophophylic), 24152105050250, GE Healthcare Life Sciences) per extraction. The beads were transferred to a $2 \mathrm{ml}$ tube and washed twice with a large volume of in $1 \%$ Tween-20. After washing, we resuspended the $10 \mu \mathrm{l}$ beads in $20 \mu \mathrm{l}$ 1\% Tween-20 and added $20 \mu \mathrm{l}$ of the Tween-20/bead mixture to each extraction (a final Tween-20 concentration of $\sim 0.02 \%$ ). DNA was precipitated onto the beads for $5-10 \mathrm{~min}$ by adding $0.7 \times$ volume of Isopropanol (420 $\mu \mathrm{l})$. We washed the magnetic beads twice with $500 \mu \mathrm{l} 80 \%$ ethanol on a magnet rack, air-dried the beads, and eluted the gDNA in $50 \mu \mathrm{l} 1 \times$ TE buffer (10 mM Tris- $\mathrm{HCl} \mathrm{pH} \mathrm{8.0,} 1 \mathrm{mM}$ EDTA) for 5-10 min. The eluted gDNA was transferred to a $1.5 \mathrm{ml}$ tube and stored at $-20^{\circ} \mathrm{C}$ until further use.

The active hands-on time without centrifugation and incubation times is $8 \mathrm{~min}$ per extraction (S1, https://doi.org/10.5281/ zenodo.4060156).

\section{7 | SDE 96-well plate gDNA cleanup}

To perform the gDNA cleanups in a 96-well format, we transferred $140 \mu \mathrm{l}$ supernatant to a 96-well plate (96 Well Non-Skirted PCR Plate, CLEAR, 4TI-0750_50, 4titude Ltd). For each extraction, we washed $5 \mu \mathrm{l}$ magnetic beads and eluted the beads in $5 \mu \mathrm{l} \%$ Tween-20 (for 96 samples, we therefore prepared $480 \mu$ magnetic beads). We added $5 \mu$ of the $1 \%$ Tween-20/bead mixture to each reaction and precipitated the DNA on the beads adding $0.7 \times$ volume of Isopropanol $(98 \mu \mathrm{l})$. We mixed the reactions by vortexing for $5 \mathrm{~s}$ and incubated the plates for $5 \mathrm{~min}$ to precipitate the gDNA. We then washed the beads twice on a magnet rack with $100 \mu \mathrm{l} 80 \%$ ethanol, removed the remaining ethanol well, and eluted the gDNA in $50 \mu \mathrm{l} 1 \times$ TE buffer for $10 \mathrm{~min}$. The cleaned gDNA was transferred to a fresh 96-well plate (96 Well Non-Skirted PCR Plate, CLEAR, 4TI0750_50, 4titude Ltd) and stored at $-20^{\circ} \mathrm{C}$ until further use.

\section{8 | Genomic soil DNA extraction and quality control}

We performed the gDNA extraction using three replicates per soil sample and extraction method, using $250 \mathrm{mg}$ soil from the same sample for each extraction. We tested four different extraction methods: (a) the PowerSoil ${ }^{\circledR}$ DNA Isolation Kit (12888-50; CAMBIO, now 12888-100, DNeasy PowerSoil, QIAGEN Ltd); (b) the MP Biomedicals ${ }^{\mathrm{TM}}$ FastDNA $^{\mathrm{TM}}$ SPIN Kit for Soil (11492400; Fisher Scientific); (c) our SDE method; and (d) the recently published paperdisk method (Zou et al., 2017). 


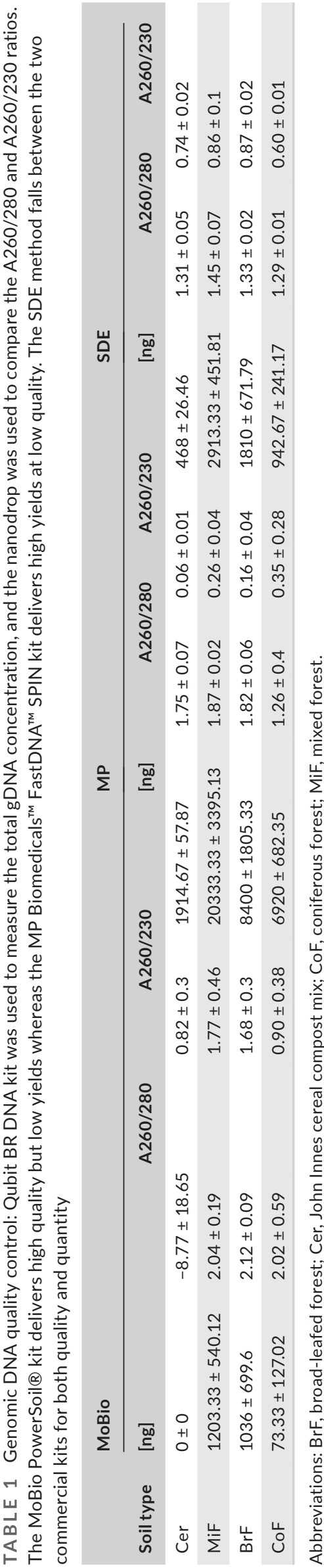

We determined the gDNA concentrations (Table 1) using the Qubit 2.0 Fluorometer (Thermo Fisher Scientific) dsDNA BR DNA assay kit (Q32853; Thermo Fisher Scientific) and assessed the purity of the extracted gDNA by measuring the 260:280 and 260:230 absorbance ratios using the NanoDrop ND-1000 Spectrophotometer (Table 1). To assess the fragment size of the extracted gDNA, we used the 2200 TapeStation (Agilent Technologies) genomic DNA screen tape (5067-5365, Agilent Technologies) and genomic DNA reagents (5067-5366; Agilent Technologies).

\subsection{Amplicon library construction, quality control, and pooling}

We targeted the bacterial variable (V4) region of the 16S rRNA gene and the fungal ITS1 region of the internal transcribed spacer (between $18 \mathrm{~S}$ and 5.8S rRNA subunit) for amplicon library construction. Amplicon library construction was performed using a two-step PCR protocol: In a first PCR, we targeted the $16 \mathrm{~S}$ V4 and ITS regions using gene-specific primers with a $5^{\prime}$ primer tail that allows the addition of the barcoded sequencing adapters for custom dual indexing in a second PCR (Giolai et al., 2019; Rowan et al., 2019; S2, https:// doi.org/10.5281/zenodo.4060156). For the bacterial 16S V4 PCRs, we included PNAs (peptide nucleic acid PCR clamps) targeting plant mitochondria (mPNA) and chloroplast regions (cPNA; Lundberg et al., 2013) in our first PCR to minimize plant gene amplification.

For the first PCR, we used the following primers adapted from Walters et al. (2016): 16S 515 forward 5'-[TCGTCGGCAGCGTC] [AGATGTGTATAAGAGACAG][GT][GTGYCAGCMGCCGCGG TAA]-3' (5'-[P5][Tn5 adapter][linker][16SV4]-3'), 16S 806 reverse $5^{\prime}$-[GTCTCGTGGGCTCGG][AGATGTGTATAAGAGACAG][CC] [GGACTACNVGGGTWTCTAAT]-3' (5'-[P7][Tn5 adapter][linker][16S V4]-3'), ITS1 forward 5'- [TCGTCGGCAGCGTC][AGATGTGTATAA GAGACAG][GG] [CT TGGTCATTTAGAGGAAGTAA] - $3^{\prime}$ (5'-[P5][Tn5 adapter][linker][ITS]-3'), and ITS2 reverse 5'-[GTCTCGT GGGCTCGG][AGATGTGTATAAGAGACAG][CG][GCTGC GTTCTTCATCGATGC]-3' (5'-[P7][Tn5 adapter][linker][ITS]-3'). All primers were ordered from Integrated DNA Technologies (IDT). The 5 ' tails of the gene $16 \mathrm{~S} \mathrm{V4}$ and ITS specific primers contain the Illumina Nextera Tn 5 transposase adapter and linker sequences. This allows further amplification of the amplicons with barcoded Illumina adapters and sequencing using Illumina chemistry with the Illumina supplied sequencing or indexing primers (all oligonucleotide sequences are in S2, https://doi.org/10.5281/zenodo.4060156).

We performed the first PCR step using $3 \mathrm{ng}$ gDNA input, 1 Unit Kapa HiFi polymerase (KK2102; Roche), 1× Kapa HiFi Fidelity buffer (KK2102; Roche), $0.25 \mu \mathrm{M}$ reverse and $0.25 \mu \mathrm{M}$ forward primer (IDT), 1× KAPA Enhancer 1 (KK5024; Sigma-Aldrich Co Ltd), 0.3 MM dNTPs (KK2102; Roche), $1.25 \mu \mathrm{M}$ cPNA (PNA BIO INC), 1 MM mPNA (PNA BIO INC), and DNase/RNase-free distilled water (10977-049; Thermo Fisher Scientific) in $10 \mu \mathrm{l}$ total reaction volume. We performed each PCR with three technical replicates using the following cycle conditions: initial denaturation at $95^{\circ} \mathrm{C}$ for $3 \mathrm{~min}$, followed by 20 cycles of denaturation at $98^{\circ} \mathrm{C}$ for 
$20 \mathrm{~s}$, PNA clamping at $75^{\circ} \mathrm{C}$ for $10 \mathrm{~s}$, primer annealing at $55^{\circ} \mathrm{C}$ for $30 \mathrm{~s}$, elongation at $72^{\circ} \mathrm{C}$ for $30 \mathrm{~s}$, with a final elongation step at $72^{\circ} \mathrm{C}$ for $3 \mathrm{~min}$ (Alpha Cycler 4, PCRmax; Labtech International Ltd). The PCR mix for the fungal libraries was the same but with ITS instead of 16S V4 primers and without the PNA oligo blockers. ITS amplification reactions were run with the following cycle conditions: initial denaturation at $95^{\circ} \mathrm{C}$ for $3 \mathrm{~min}$, followed by 20 cycles of denaturation at $98^{\circ} \mathrm{C}$ for $20 \mathrm{~s}$, annealing at $55^{\circ} \mathrm{C}$ for $30 \mathrm{~s}$, elongation at $72^{\circ} \mathrm{C}$ for $30 \mathrm{~s}$, with a final elongation at $72^{\circ} \mathrm{C}$ for 3 min (Alpha Cycler 4, PCRmax; Labtech International Ltd.).

After this first gene targeting PCR step, we pooled the three technical replicates reactions for the same sample and conducted a $0.7 \times$ magnetic bead cleanup (HighPrep ${ }^{\mathrm{TM}}$ PCR Clean-up System, AC-60050, MAGBIO). The clean PCR products were eluted in $10 \mu \mathrm{l}$ $1 \times$ TE buffer. A second PCR was conducted on the pooled replicate sample with the same program for bacterial and fungal libraries, that is, using 1× Kapa HiFi Fidelity buffer (KK2102; Roche), 1 Unit Kapa HiFi polymerase (KK2102; Roche), $0.2 \mu \mathrm{M}$ P5 indexing primer (IDT), $0.2 \mu \mathrm{M}$ P7 indexing primer (IDT), $0.3 \mu \mathrm{M}$ dNTPs (KK2102; Roche), and $7.6 \mu \mathrm{l}$ of clean gene targeting PCR product and DNase/RNasefree distilled water (10977-049; Thermo Fisher Scientific) in a total reaction volume of $30 \mu \mathrm{l}$. The barcoding cycle conditions (Alpha Cycler 4, PCRmax; Labtech International Ltd.) were as follows: initial denaturation at $95^{\circ} \mathrm{C}$ for $3 \mathrm{~min}$, followed by 15 cycles of denaturation at $98^{\circ} \mathrm{C}$ for $20 \mathrm{~s}$, annealing at $62^{\circ} \mathrm{C}$ for $30 \mathrm{~s}$, elongation at $72^{\circ} \mathrm{C}$ for $30 \mathrm{~s}$, with a final elongation at $72^{\circ} \mathrm{C}$ for $3 \mathrm{~min}$. After the barcoding $\mathrm{PCR}$ step, the reactions were cleaned using a $0.7 \times$ magnetic bead cleanup (HighPrep ${ }^{\mathrm{TM}}$ PCR Clean-up System, AC-60050, MAGBIO) and the final libraries eluted in $20 \mu \mathrm{l}$ EB buffer ( $10 \mathrm{mM}$ Tris- $\mathrm{HCl} \mathrm{pH}$ 8.5).

We quantified the cleaned amplicon libraries using the Qubit 2.0 Fluorometer (Thermo Fisher Scientific) with dsDNA HS Assay Kit reagents (Q32854; Thermo Fisher Scientific) and controlled the size of the amplicons on the GX Touch using the $3 \mathrm{~K}$ kit (X-Mark DNA LabChip, CLS144006, HT DNA NGS 3 K Reagent Kit, Perkin Elmer LAS (UK) LTD). The amplicons were pooled equimolarly to $1.5 \mathrm{nM}$ for bacterial and $2.0 \mathrm{nM}$ for fungal libraries according to the molarity obtained by the LabChip GX Touch smear analysis of the region between 380 and $650 \mathrm{bp}$. We performed a final $0.7 \times$ magnetic bead cleanup (HighPrep ${ }^{\mathrm{TM}}$ PCR Clean-up System, AC-60050, MAGBIO) of the libraries and eluted the final library pools in $50 \mu \mathrm{l}$ EB buffer.

\subsection{0 | Sequencing}

The $16 \mathrm{~S}$ and ITS library pools were sequenced using the MiSeq Nano reagent version 2, 500 cycle kit (Illumina) at an 8 pM loading concentration with a $10 \%$ PhiX spike-in. $16 \mathrm{~S}$ and ITS pools were sequenced separately-each pool using a MiSeq Nano reagent kit.

\subsection{1 | Amplicon data analysis}

We demultiplexed bcl files using bcl2fastq version 2-2.20.0.422 with the settings-barcode-mismatches 1-fastq-compression-level 9 into individual fastq.gz files. We trimmed the paired-end reads for primers, sequencing adapters and linker sequences using cutadapt-1.9.1 (Martin, 2011) with the settings $-n$ 4-minimum-length $=50$. The data were quality controlled using R-3.5.0 and DADA2 version 1.8.0 according to the workflow described in (Callahan et al.,) version 2 . The truncation length for forward reads was set to $180 \mathrm{bp}$ and the truncation length for the reverse reads to $200 \mathrm{bp}$. For $16 \mathrm{~S}$ libraries, we used the following parameters: $\max N=0, \operatorname{maxEE}=2$ and truncQ $=11$. For ITS libraries, we specified the following parameters: $\operatorname{maxN}=0, \operatorname{maxEE}=c(2,2)$ and truncQ $=11$ and a minimum length of $50 \mathrm{bp}$. Forward and reverse reads were merged with default settings. We used the Silva (silva_nr_v132) database to classify bacterial reads (Quast et al., 2013) and UNITE (sh_general_release_ dynamic_s_01.12.2017) for the fungal dataset (Nilsson et al., 2019). Reads that did not match to the bacterial or fungal database were removed. Alpha-diversity analysis (Shannon and Observed measure) was performed on pre-normalized data (package "phyloseq," R-3.5.0, version 2.5.2). For beta-diversity analysis, ASVs with a mean lower as $10^{-5}$ were removed from the datasets. The filtered data with 4113 bacterial ASVs and 1602 fungal ASVs (package "phyloseq," version 1.24.0) were used to calculate the $\beta$-diversity (Bray-Curtis, R-3.5.0 "vegan" package, version 2.5.2) and to perform statistical analysis (package "vegan," ANOSIM and PERMANOVA: adonis function; Dixon, 2003). All numbers of processed reads through the analysis pipeline are in S3, https://doi.org/10.5281/zenodo.4060156.

We performed the correlation analysis in R-3.5.0 using the filtered phyloseq object on genus level and plotted it with log10 scaling (McMurdie \& Holmes, 2013). The corrplot was generated in R, using the filtered phyloseq object on order level with the corrplot package version 0.84. All figures were generated in R-3.5.0 using the R package ggplot2-3.1.1 (Ginestet, 2011).

\section{3 | RESULTS}

\subsection{DNA yield and fragment analysis of different extraction methods}

We tested our SDE method by extracting gDNA from $250 \mathrm{mg}$ samples of four different soil types taken from a mixed forest (MiF), a coniferous forest (CoF), and a broad-leafed forest (BrF), plus a standardized cereal crop compost mix used at the John Innes Centre (Cer). We compared our method to two frequently used commercial extraction kits: MP Biomedicals ${ }^{\mathrm{TM}}$ FastDNA ${ }^{\mathrm{TM}}$ SPIN and MoBio PowerSoil ${ }^{\circledR}$ and a recently published low-cost paperdisk method described to extract microbial DNA suitable for PCR in less than $30 \mathrm{~s}$ (Zou et al., 2017). We first determined which gDNA extraction method produces the highest yield and best gDNA quality using fluorometric and spectrophotometric analysis. The MP Biomedicals ${ }^{\mathrm{TM}}$ FastDNA $^{\mathrm{TM}}$ SPIN kit delivered the highest and the MoBio PowerSoil ${ }^{\circledR}$ kit the lowest gDNA yield (Table 1). The highest gDNA purity (A260/A280 and A260/A230 ratios) was obtained by the MoBio PowerSoil ${ }^{\circledR}$ kit and the lowest by the MP Biomedicals ${ }^{\mathrm{TM}}$ FastDNA ${ }^{\mathrm{TM}}$ SPIN kit (Table 1). Our method scored between the two commercial kits for both quality and quantity (Table 1). We further evaluated the methods for the 
extracted gDNA fragment length on the Agilent TapeStation. For the MoBio PowerSoil ${ }^{\circledR}$ kit method, we found the majority of gDNA fragment sizes fall between 13.9 and $24.4 \mathrm{~kb}$. The SDE method produced fragments centered between 11.3 and $11.7 \mathrm{~kb}$ and the MP Biomedicals $^{\mathrm{TM}}$ FastDNA $^{\mathrm{TM}}$ SPIN mostly extracted fragments below $10 \mathrm{~kb}$ (Figure A2). For the paperdisk method, we could not obtain enough DNA for fragment analysis.

(a) $16 \mathrm{~S}$ MoBio

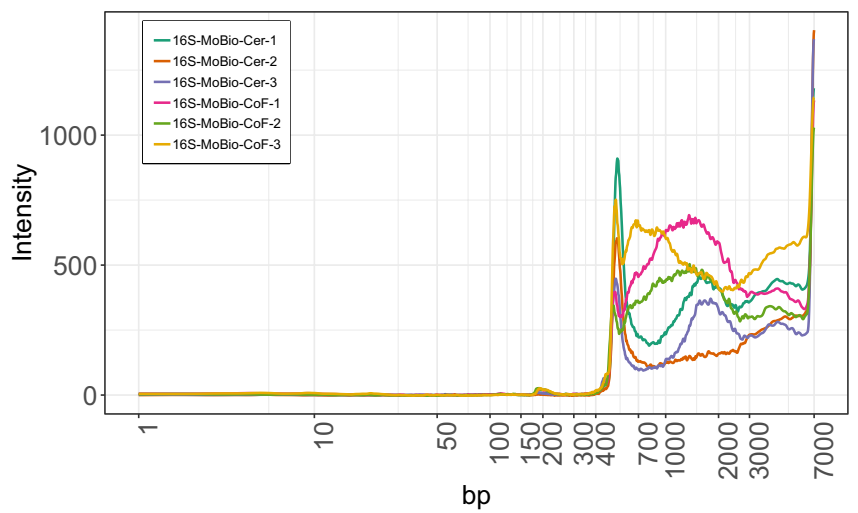

(c) 16S SDE

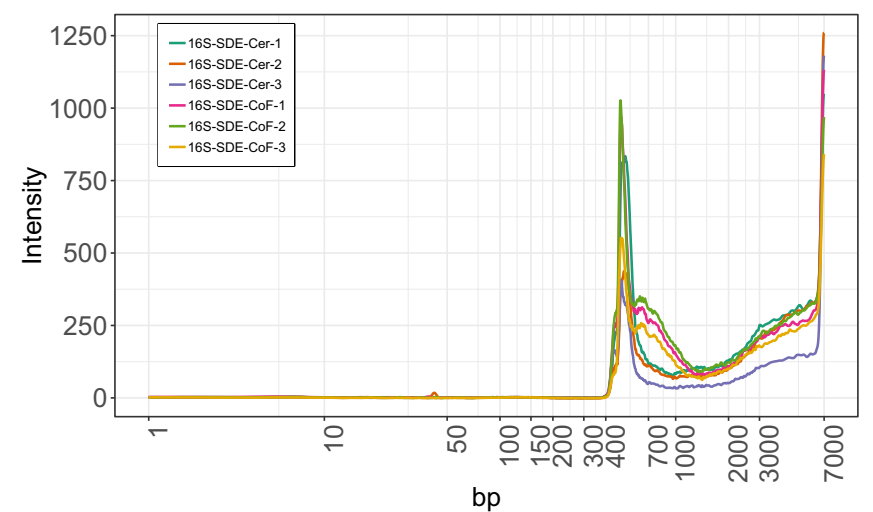

(e) $16 S M P$

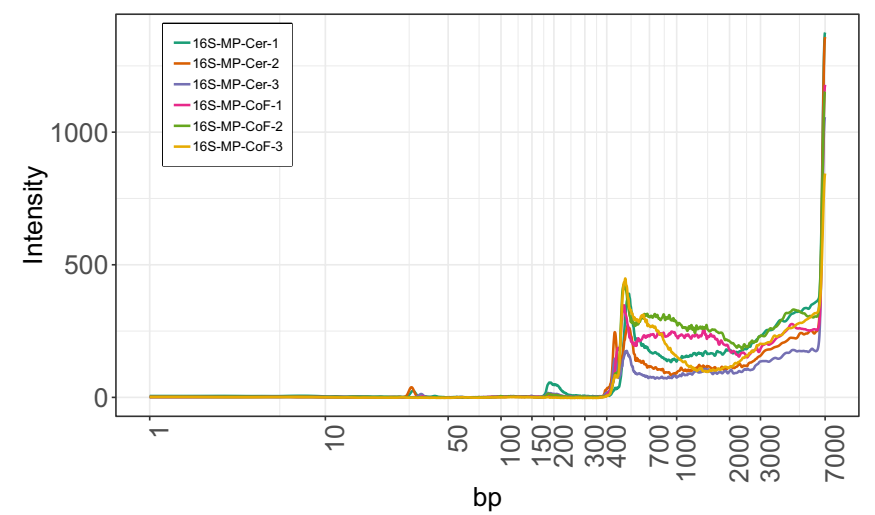

\subsection{Extraction method effects on bacterial and fungal amplicon library construction}

We constructed 16S V4 and ITS rRNA Illumina sequencing libraries from all extractions (three biological replicates per soil type) using $3 \mathrm{ng}$ of gDNA input per library construction reaction and three technical replicates (similar to Tourlousse et al., 2018). All libraries were

\section{(b) ITS MoBio}

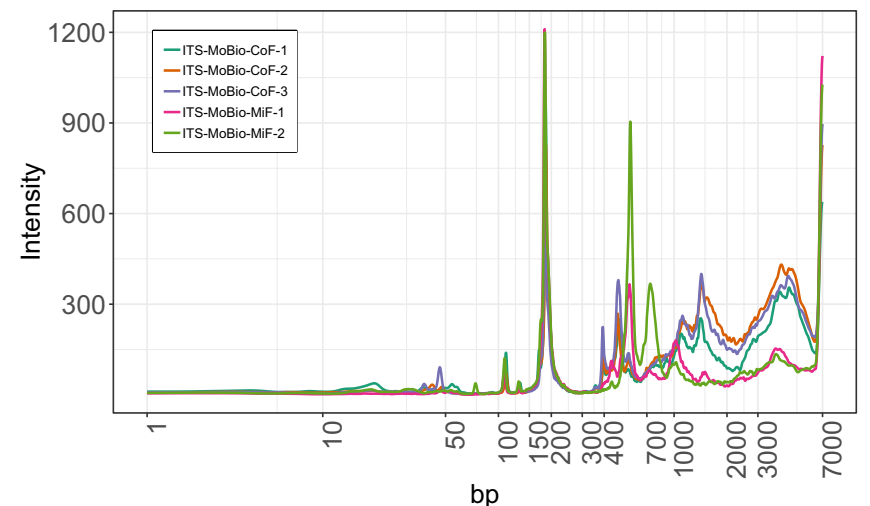

(d) ITS SDE

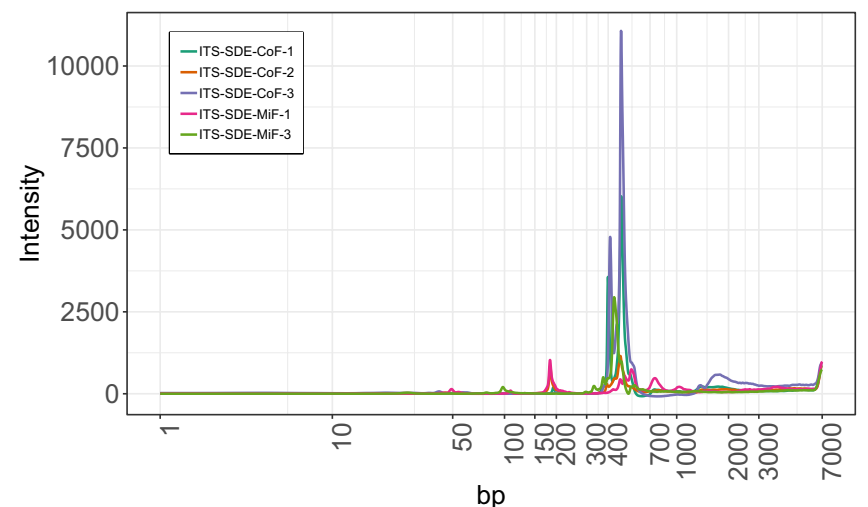

(f) ITS MP

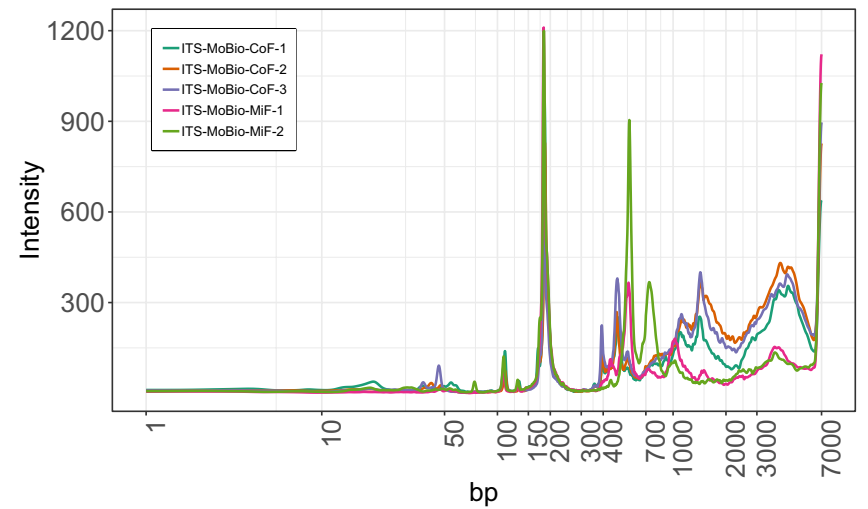

FIG URE 2 Representative results comparing MoBio PowerSoil ${ }^{\circledR}$, MP Biomedicals ${ }^{\mathrm{TM}}$ FastDNA ${ }^{\mathrm{TM}}$ SPIN and SDE methods run on a LabChip GX Touch: (a) Representative $16 S$ libraries of gDNA extracted with MoBio PowerSoil ${ }^{\circledR}$. (b) Representative ITS libraries of gDNA extracted with MoBio PowerSoil ${ }^{\circ}$. (c) Representative 16S libraries of gDNA extracted with SDE. (d) Representative ITS libraries of gDNA extracted with SDE. (e) Representative $16 \mathrm{~S}$ libraries of extracted gDNA with MP Biomedicals ${ }^{\mathrm{TM}}$ FastDNA $^{\mathrm{TM}}$ SPIN. (f) Representative ITS libraries of extracted gDNA with MP Biomedicals ${ }^{\mathrm{TM}}$ FastDNA $^{\mathrm{TM}}$ SPIN. The target size of representative $16 \mathrm{~S}$ libraries is between 350 and 500 bp and for ITS libraries between 300 and 700 bp. gDNA, genomic DNA; SDE, soil gDNA extraction method 
TABLE 2 Correlation coefficient between different extraction methods and on four different soil types for fungal and bacterial ASVs: Correlation coefficient between different gDNA extraction method derived ASVs for all extraction method combinations for fungal and bacterial data (SDE/MoBio, SDE/MP, and MoBio/MP)

\begin{tabular}{|c|c|c|c|}
\hline & Soil type & Extraction method & $R$ \\
\hline $16 S$ & Cer & SDE/MoBio & .75 \\
\hline $16 S$ & $\mathrm{BrF}$ & SDE/MoBio & .74 \\
\hline $16 S$ & $\mathrm{CoF}$ & SDE/MoBio & .94 \\
\hline $16 S$ & $\mathrm{MiF}$ & SDE/MoBio & .85 \\
\hline $16 \mathrm{~S}$ & Cer & $\mathrm{MP} / \mathrm{SDE}$ & .84 \\
\hline $16 S$ & $\mathrm{BrF}$ & $\mathrm{MP} / \mathrm{SDE}$ & .6 \\
\hline $16 S$ & $\mathrm{CoF}$ & MP/SDE & .9 \\
\hline $16 S$ & $\mathrm{MiF}$ & MP/SDE & .73 \\
\hline $16 S$ & Cer & MP/MoBio & .79 \\
\hline $16 S$ & $\mathrm{BrF}$ & MP/MoBio & .79 \\
\hline $16 S$ & $\mathrm{CoF}$ & MP/MoBio & .9 \\
\hline $16 S$ & MiF & MP/MoBio & .76 \\
\hline ITS & Cer & SDE/MoBio & .88 \\
\hline ITS & $\mathrm{BrF}$ & SDE/MoBio & .49 \\
\hline ITS & $\mathrm{CoF}$ & SDE/MoBio & .85 \\
\hline ITS & MiF & SDE/MoBio & .86 \\
\hline ITS & Cer & MP/SDE & .8 \\
\hline ITS & $\mathrm{BrF}$ & $\mathrm{MP} / \mathrm{SDE}$ & .49 \\
\hline ITS & $\mathrm{CoF}$ & $\mathrm{MP} / \mathrm{SDE}$ & .82 \\
\hline ITS & MiF & $\mathrm{MP} / \mathrm{SDE}$ & .82 \\
\hline ITS & Cer & MP/MoBio & .89 \\
\hline ITS & $\mathrm{BrF}$ & MP/MoBio & .86 \\
\hline ITS & $\mathrm{CoF}$ & MP/MoBio & .88 \\
\hline ITS & $\mathrm{MiF}$ & MP/MoBio & .86 \\
\hline
\end{tabular}

Abbreviations: BrF, soil from a broad-leafed forest; Cer, standard cereal crop soil mix used at JIC, Norwich, UK; CoF, soil from a coniferous forest; MiF, soil from a mixed forest. inspected using LabChip GX Touch high-sensitivity capillary electrophoresis. The gDNA extracted with MP Biomedicals ${ }^{\mathrm{TM}}$ FastDNA ${ }^{\mathrm{TM}}$ SPIN, MoBio PowerSoil ${ }^{\circ}$, and our SDE method performed well in library construction, producing libraries with similar profiles (Figure 2). The paperdisk method did not produce a library with a detectable electropherogram trace. We pooled all libraries at equal mass (except the paperdisk method where we used the full amount as these libraries were not detectable) and submitted each library for $250 \mathrm{bp}$ paired-end sequencing. Sequencing of the paperdisk extraction method did not produce any reads suggesting that the extracted gDNA concentration was too low for successful library construction.

\subsection{Comparison of extraction methods based on bacterial and fungal microbial composition}

It has been previously reported that different microbial gDNA extraction methods can introduce a genera bias (Sáenz et al., 2019). To test this, we compared the biological replicates of each library preparation method (apart from the paperdisk method) by correlation analysis of the detected bacterial (total of 4069) and fungal (total of 1549) amplicon sequence variants (ASVs; Table 2, Figure A3). The three tested gDNA extraction methods compared well across all soil types. We also compared the genus abundances of our SDE method with the two commercial kits by analyzing the bacterial and fungal genus abundances of each library. The genus abundance plots for each soil type were not statistically significantly different between the extraction methods used for either fungal (adonis test, $p$-value: .801 , Table 3) or bacterial communities (adonis test, p-value: 0.579 , Table 3). Instead, our data showed statistically significant variation between soil types (bacteria ANOSIM test, $p$-value: $9.99 \mathrm{e}-4$, fungi ANOSIM test, p-value: $9.99 \mathrm{e}-4$, Table 3) but not between gDNA extraction methods (bacteria: Figure $3 a, b$ and fungi: Figure $3 c, d$ ). We further tested the samples using beta diversity as a measure

TAB LE 3 Statistical results of alpha- and beta-diversity analysis: Bacterial and fungal data were investigated for potential soil type and gDNA extraction method influence on alpha and beta diversity. For alpha diversity, Shannon diversity and Observed richness were used, and for beta diversity, Bray-Curtis distance matrix was used.

\begin{tabular}{|c|c|c|c|c|c|c|c|}
\hline & Question & \multicolumn{3}{|c|}{ Alpha diversity } & \multicolumn{3}{|c|}{ Beta diversity } \\
\hline $16 S$ & Soil type & Shannon & & 0.018507038 & & & \\
\hline $16 S$ & Soil type & Observed & 0.032518656 & & & & \\
\hline $16 S$ & Extraction method & Shannon & & 0.466618041 & & & \\
\hline $16 S$ & Extraction method & Observed & 0.365539882 & & & & \\
\hline $16 S$ & Extraction method & & & & Bray-Curtis & & 0.579 \\
\hline ITS & Soil type & Observed & 0.103862 & & & & \\
\hline ITS & Soil type & Shannon & 0.214273 & & & & \\
\hline ITS & Soil type & & & & Bray-Curtis & 0.000999001 & \\
\hline ITS & Extraction method & Shannon & 0.018627631 & & & & \\
\hline
\end{tabular}


(a) 16S Genus level soil: Top 30

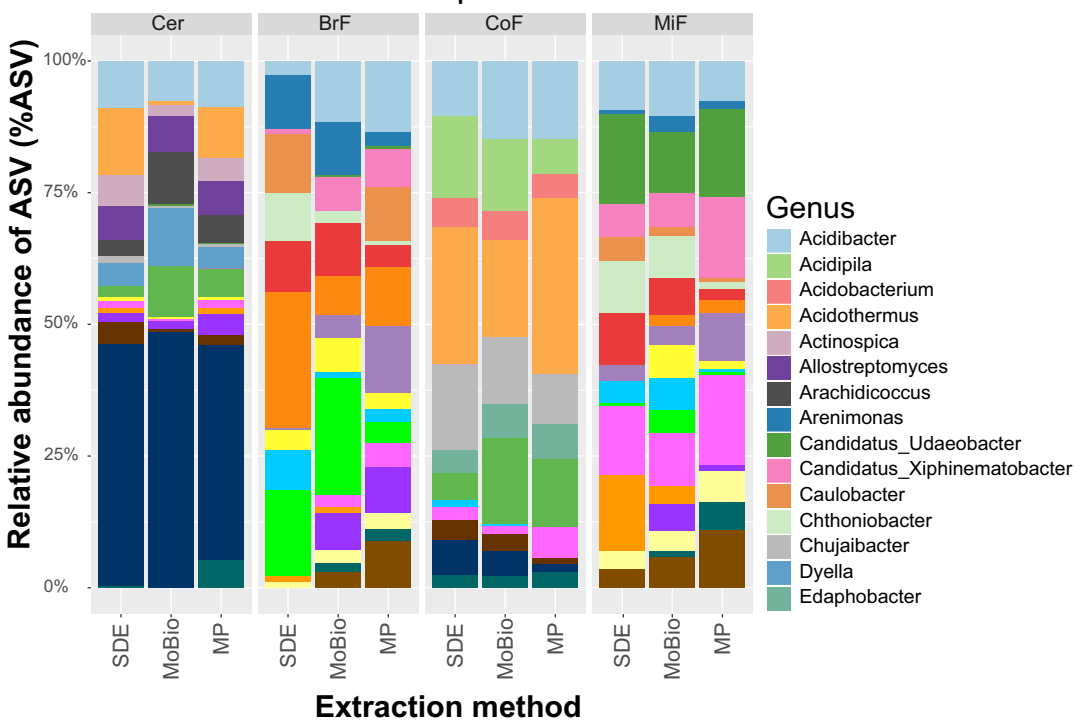

(c) ITS Genus level soil: Top 30

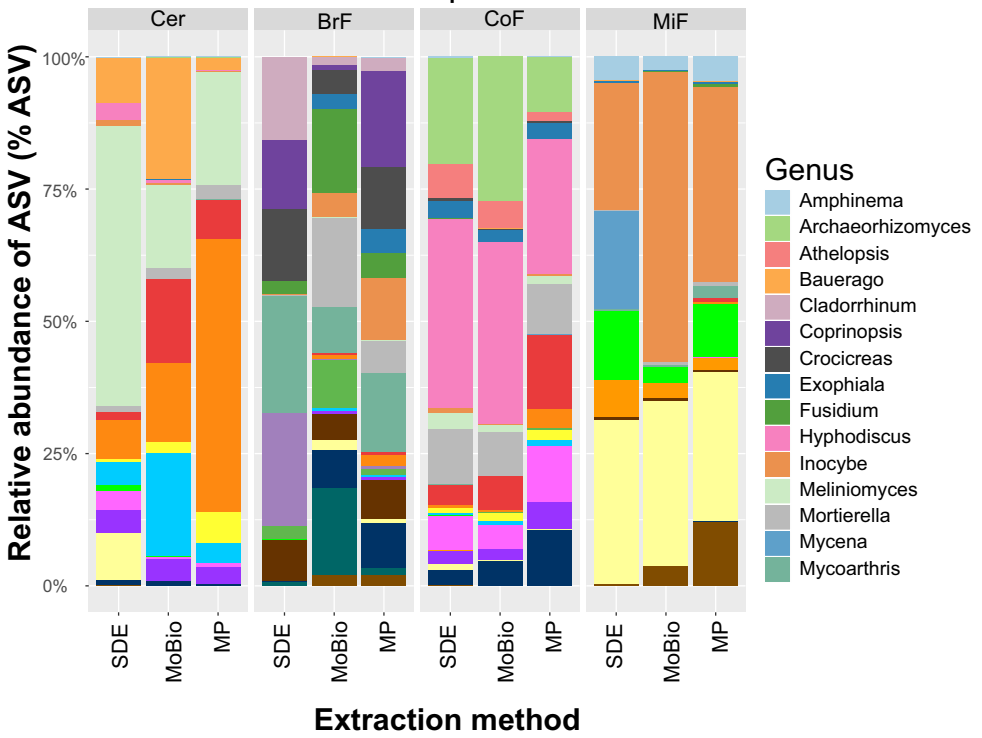

(b)

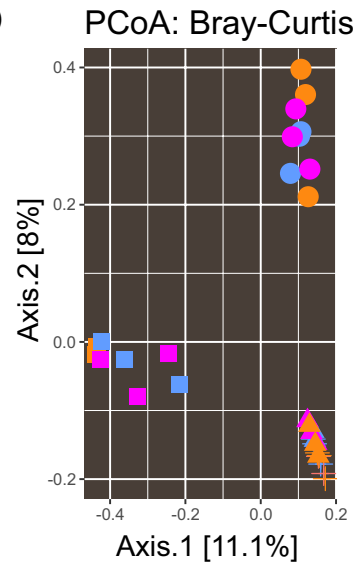

extraction method location

$\begin{array}{ll}\text { SDE } & \text { Cer } \\ \text { MoBio } & \mathbf{B r F} \\ \text { MP } & \square \mathrm{CoF} \\ & +\mathrm{MiF}\end{array}$

(d) PCoA: Bray-Curtis

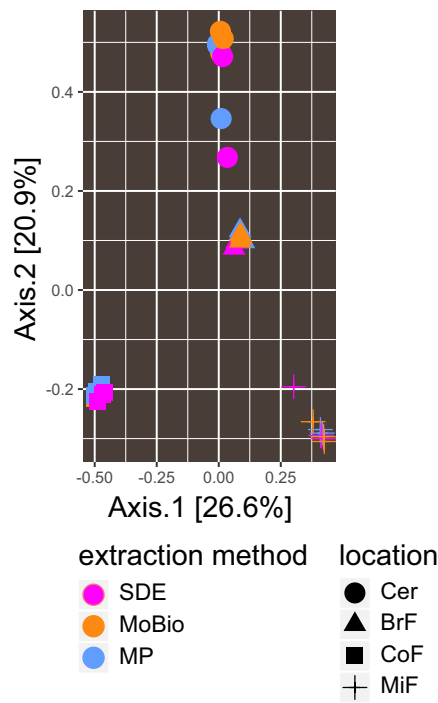

FIGURE 3 PCoA comparison of ASV abundances and relative abundance bar chart for bacterial and fungal communities of three different gDNA extraction methods and four soil types: (a) Top 30 bacterial community composition representing $0.72 \%$ of the overall bacterial community. (b) PCoA showing beta diversity by Bray-Curtis distance for bacterial community composition. (c) Top 30 fungal community composition representing $1.87 \%$ of the overall fungal community. (d) PCoA showing beta diversity by Bray-Curtis distance for fungal community composition. (a) and (b) show that the clustering for the entire bacterial communities is soil type-dependent and not driven by different gDNA extraction methods. (c) and (d) show the same is true for the fungal community structure. Statistical analysis shows no significant difference between DNA extraction methods used for both bacterial (adonis test, $p$-value: .579) and fungal (adonis test, $p$-value: .801) communities, but shows a significant difference between locations for bacterial (ANOSIM test, $p$-value: 9.99e-4) and fungal (ANOSIM test, $p$-value: 9.99e-4) communities. ASVs, amplicon sequence variants; BrF, broad-leafed forest soil; Cer, standard cereal compost used at the JIC; CoF, coniferous forest soil; MiF, mixed forest soil; SDE, soil gDNA extraction method, MoBio, MoBio PowerSoil@; MP, MP Biomedicals ${ }^{\mathrm{TM}}$ FastDNA $^{\mathrm{TM}}$ SPIN

(Bray-Curtis) for between-sample similarity. This analysis agreed with the result of the genus abundance plots, that is, by clustering the soil types separately but not clustering the data for the three extraction methods (Figure 3b,d). We confirmed this result with permutation multivariate analysis of variance analyses (PERMANOVA, package "vegan" version 2.5.2, adonis function). We also controlled the impact of gDNA extraction methods on alpha diversity (Figure 4). We could not find an impact of the gDNA extraction method on the bacterial alpha diversity (Shannon diversity ANOVA test, p-value: 0.466618, Observed richness Kruskal test, p-value: 0.36554), but observed that bacterial alpha-diversity differences are driven by soil type (Table 3, Shannon diversity ANOVA test, p-value: 0.018507, 


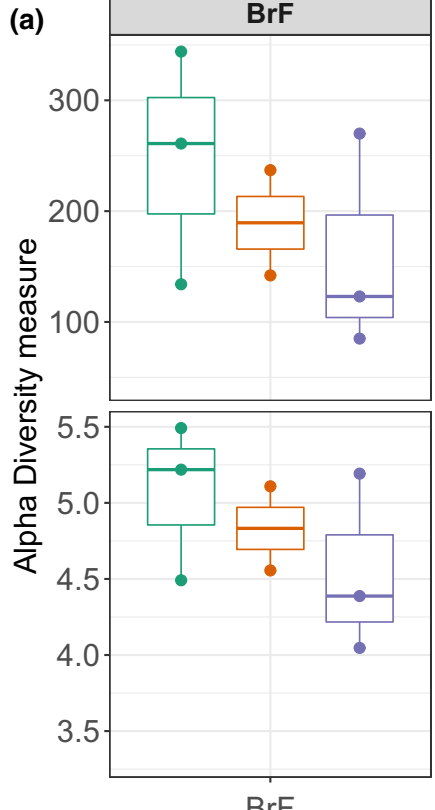

$\mathrm{BrF}$

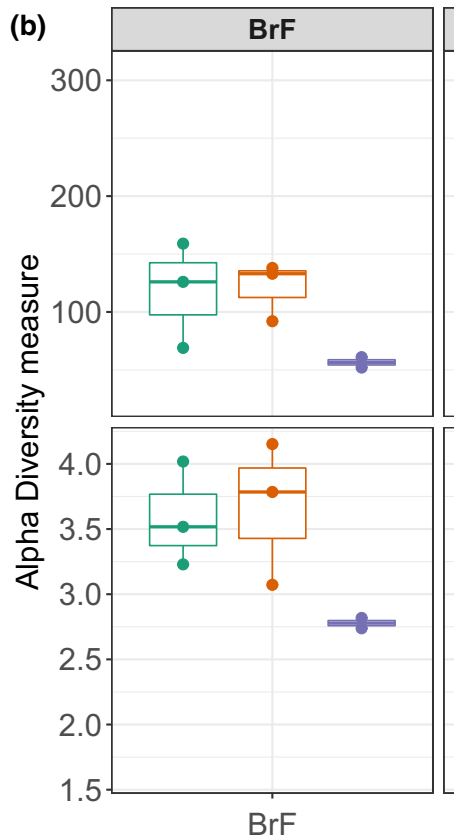

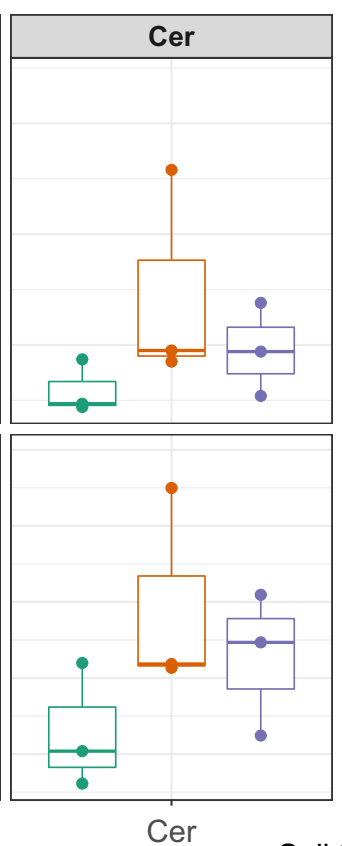

Soil type
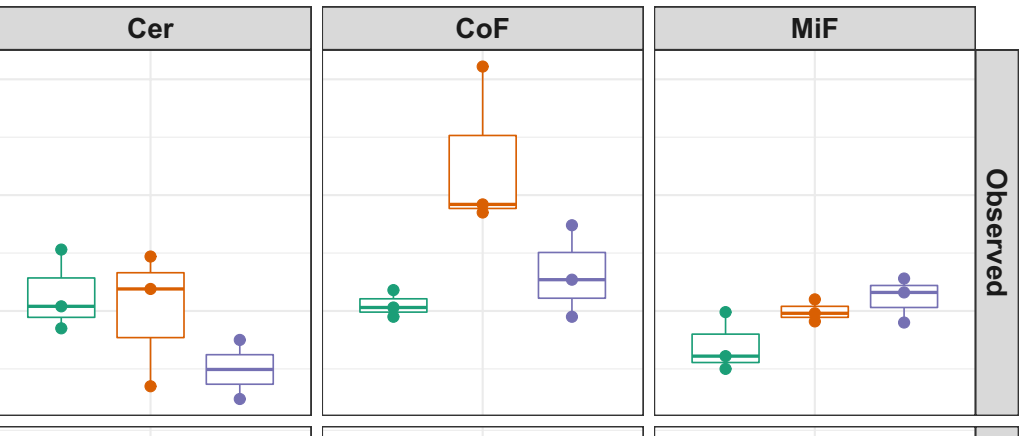

\begin{tabular}{l} 
음 \\
$\frac{0}{0}$ \\
$\frac{0}{2}$ \\
\hdashline \\
$\stackrel{2}{2}$
\end{tabular}

\section{Extraction method}

由oBio

MP

\$ SDE

FIGURE 4 Alpha-diversity comparison for bacterial and fungal communities of three different gDNA extraction methods and four soil types: (a) Bacterial alpha-diversity overview of the observed richness and Shannon diversity. No influence on alpha diversity by extraction method (Shannon diversity ANOVA test, $p$-value: .466618, observed richness Kruskal test, $p$-value: .36554), but by soil type (Shannon diversity ANOVA test, $p$-value: .018507, Observed richness Kruskal test, $p$-value: .032519). No influence by soil type on fungal alpha diversity (Shannon diversity Kruskal test, $p$-value: .21473, observed diversity Kruskal test, $p$-value: .103862) and some influence of extraction method (Shannon diversity Kruskal test, $p$-value: .018628, observed richness Kruskal test, $p$-value: .168873). BrF, broad-leafed forest soil; Cer, standard cereal compost used at the JIC; CoF, coniferous forest soil; gDNA, genomic DNA; MiF, mixed forest soil; MoBio,

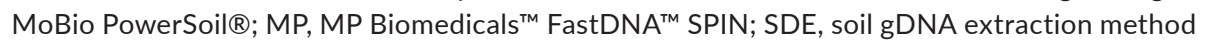

Observed richness Kruskal test, p-value: 0.032519). Fungal alpha diversity does not show a significantly different effect due to soil type (Table 3, Shannon diversity Kruskal test, $p$-value: .21473, Observed richness Kruskal test, $p$-value: .103862) and only minor differences of the gDNA extraction method (Shannon diversity Kruskal test, $p$-value: .018628, Observed richness Kruskal test, $p$-value: .168873). To compare the extraction methods in more detail and study any potential ASV-related bias, we compared the ASV abundances of each kit with the abundances assessed with our method. In the SDE to MoBio PowerSoil ${ }^{\circledR}$ kit comparison, we found the following 
(a) $16 \mathrm{~S}$

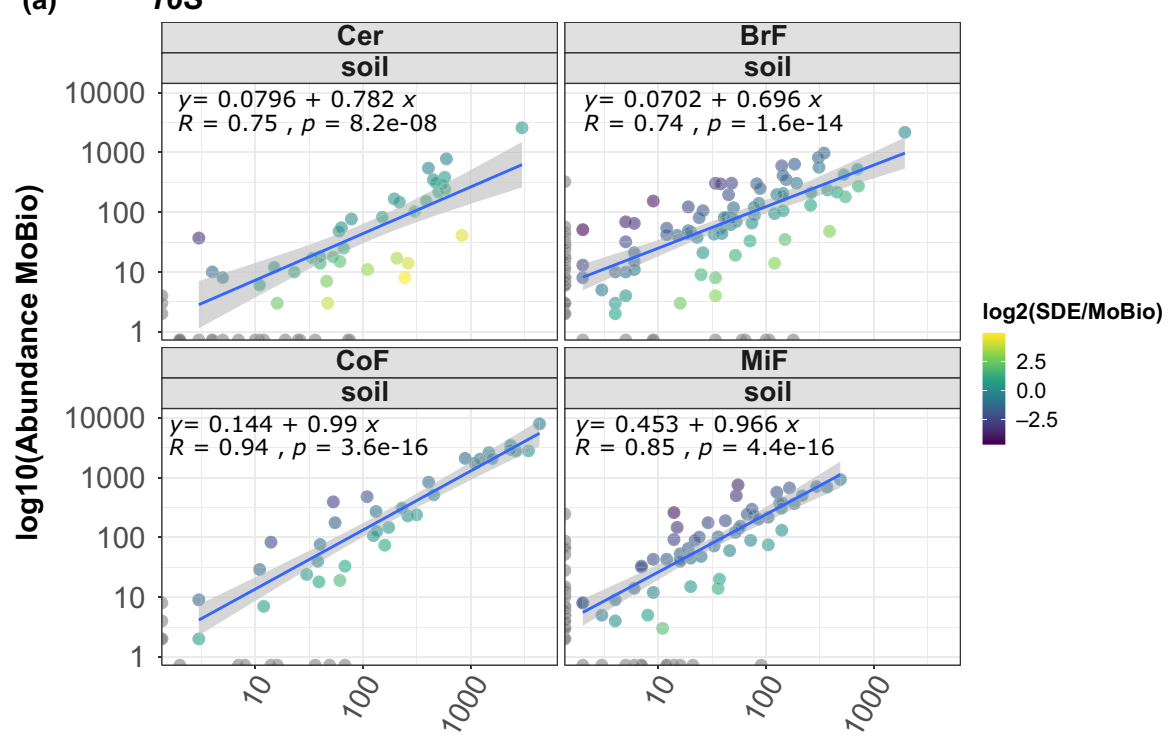

$\log 10$ (Abundance SDE)

(b) ITS

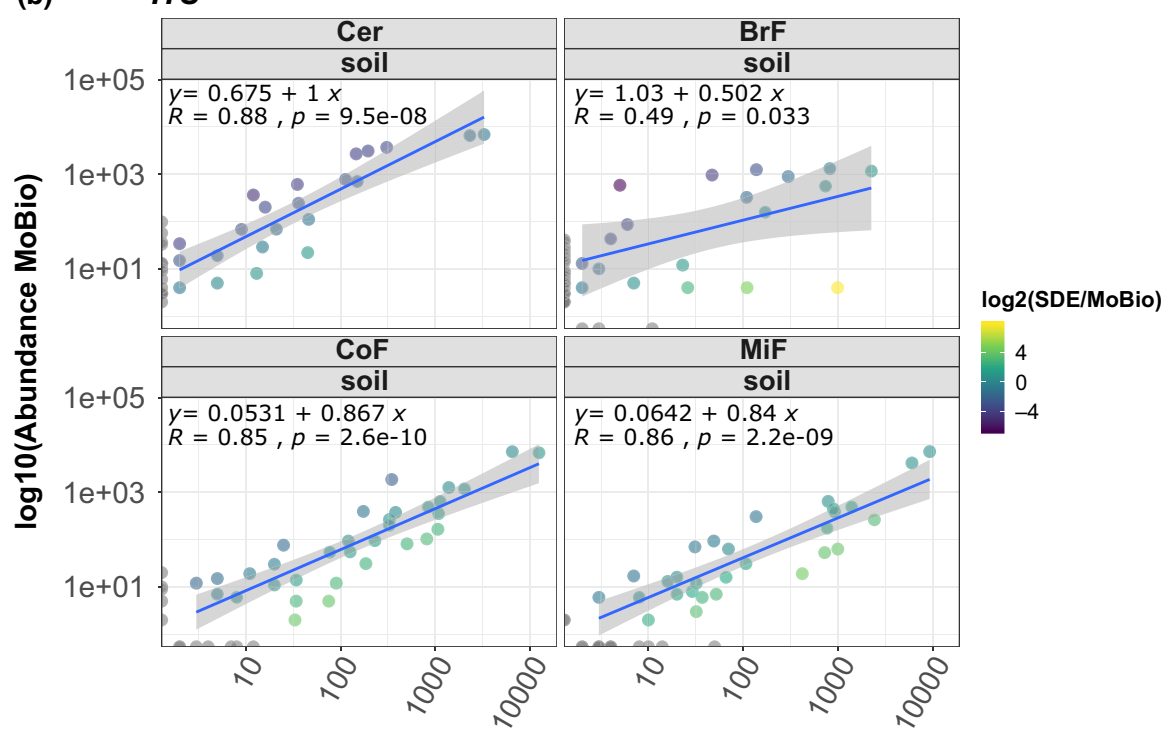

log10(Abundance SDE)

FIGURE 5 Correlation analysis of bacterial and fungal ASVs from SDE versus MoBio PowerSoil@ gDNA extraction: (a) shows bacterial ASV correlation between MoBio PowerSoil ${ }^{\circledR}$ and SDE for four different soil types. (b) shows fungal ASV correlation between MoBio PowerSoil ${ }^{\circledR}$ and SDE for four different soil types. Both extraction methods led to bacterial and fungal ASV that show a positive correlation, indicating that there is no extraction method-induced bias regarding sequenced ASV. Abundances on $x$ and $y$ axes were log10 scaled, each dot represents a genus, and dots are colored as log2(Abundance[SDE]/Abundance[Commercial Kit]). Gray dots on each axis are Genera uniquely detected for the extraction method. ASV, amplicon sequence variants; BrF, soil from a broad-leafed forest; Cer, standard cereal crop soil mix used at JIC; CoF, soil from a coniferous forest; MiF, soil from a mixed forest

correlation coefficients for bacterial ASVs over the different soil types: Cer 0.75, BrF 0.74, CoF 0.94, MiF 0.85 (Figure 5a; Table 2) and for fungal ASVs: Cer 0.88, BrF 0.49, CoF 0.85, MiF 0.86 (Figure 5b; Table 2). The correlation analysis of the SDE to MP Biomedicals ${ }^{\mathrm{TM}}$ FastDNA $^{\mathrm{TM}}$ SPIN kit delivered similar results (Cer 0.84, BrF 0.6, CoF 0.9, MiF 0.73 for bacteria, Figure $\mathrm{A} 4 \mathrm{a}$ and Cer 0.8, BrF 0.49, CoF 0.82, MiF 0.82 for fungi, Figure A4c; Table 2). These results confirm that our SDE method fits between two commonly used commercial soil gDNA extraction kits across a broad range of measurable parameters.

\section{4 | DISCUSSION}

Custom gDNA extraction methods for soil samples have been described previously (Bürgmann et al., 2001; Fatima et al., 2014; Robe 
et al., 2003; Verma et al., 2017; Yeates et al., 1998; Zhou et al., 1996). These methods emphasized gDNA quantity (Bürgmann et al., 2001; Fatima et al., 2014), quality (Bürgmann et al., 2001; Fatima et al., 2014; Verma et al., 2017), or cost-efficiency (Devi et al., 2015; Fatima et al., 2014; Yeates et al., 1998; Zou et al., 2017). However, an often overlooked but practically important consideration is the hands-on time required per extraction without a quantity or quality penalty. KatharoSeq (Minich, Zhu, et al., 2018), for example, is a pipeline for low biomass samples that delivers good gDNA quality with less hands-on time; however, it still uses parts of a commercial kit, which increases the price per sample. On the other hand, Zou et al. described a fast and very affordable gDNA extraction method but yielding lower gDNA quantities (Zou et al., 2017). Here, we present a high-throughput gDNA extraction method that is suitable for low input and inhibitor-rich sample types such as soils (Figure 1; Figure A1). For our method, we first optimized mechanical lysis conditions by increasing the amount and types of grinding material, then chemical additives to the lysis buffer which remove common contaminants. We also compared commercial kits (silica column and carboxylate-coated beads) with our carboxylated magnetic bead-based protocol to determine best extraction yields. For the additives, we used sodium phosphate as a buffer matrix, which in the past together with aluminum ions was recommended for efficient removal of humic acids while minimizing DNA losses during extraction (Mandalakis et al., 2018). We found that adding aluminum sulfate in the lysis buffer leads to increased DNA yields and purity. Although we successfully extracted DNA adding only aluminum sulfate, we also observed that the addition of ammonium acetate to further precipitate impurities (Yu \& Mohn, 1999) increased PCR amplification success (S4, https://doi. org/10.5281/zenodo.4060156 for more experimental details). We compared our finalized protocol to two leading commercial (MoBio PowerSoil ${ }^{\circledR}$ and MP Biomedicals ${ }^{\mathrm{TM}}$ FastDNA $^{\mathrm{TM}}$ SPIN kits) and a non-commercial (paperdisk based) extraction method (Zou et al., 2017) and observed that our SDE method delivered good quality and quantities of NGS-compatible gDNA at a fraction of the cost of commercially available solutions. We also find that our method requires less manually intensive centrifugation steps (MoBio: nine steps, MP: nine steps, SDE: three steps) and the total hands-on time of our method is lower than the hands-on times for the MoBio and MP kits (MoBio: $16 \mathrm{~min}$, MP: $10 \mathrm{~min}, \mathrm{SDE}: 8 \mathrm{~min}$ ). We achieved this with the use of a modified SPRI bead extraction protocol that allows fast, scalable, and inexpensive extraction of nucleic acids (Oberacker et al., 2019), especially because magnetic particles enable the transfer of our protocol to a plate format, without the disadvantages of handling many tubes and minimizing potential sample mix-ups (S1, https://doi.org/10.5281/zenodo.4060156). We tested our extraction method for 96-well plate compatibility by quantifying yields from 27 different soil types of 196 samples (Figure A1). Because it uses simple pipetting steps, SPRI beadbased purification and washing steps, our method should be easily adaptable from a multi-channel pipette to common liquid handling robotic systems (typically already able to use bead-based methods for DNA and RNA NGS library construction). The extracted gDNA from four distinctively different soil types using SDE is similar in quality and quantity to the two commercial kits (Table 1), with the extracted gDNA from the commercial kits and the SDE method led to similar amplicon library profiles (Figure 2). In contrast, the paperdisk method did not generate useable sequencing libraries. We further investigated the gDNA preparation methods for extraction biases when analyzing fungal and bacterial communities. Here, the results of the two commercial kits and the SDE method overlap, showing no statistical differences in microbiome composition (Figure 3 ) for beta diversity. Clustering of the sequencing data using PCoA separated soil types but not gDNA extraction methods (Figure 3b,d). A correlation analysis between SDE and MoBio, SDE and MP, and MoBio and MP, for detected bacterial and fungal communities (Figure 5; Figures $\mathrm{A} 3$ and $\mathrm{A} 4$ ), showeda strong correlation between the commercial kits and the SDE method the one exception being the fungal BrF samples. We suspect that the lower correlation of 0.49 between SDE and MoBio and SDE and MP for the fungal BrF soil samples (Table 3 ) could potentially be due lower read depth of the SDE BrF samples compared to the other samples (S3, https://doi.org/10.5281/zenodo.4060156). Further, bacterial alpha-diversity analysis for Shannon diversity index and Observed richness is not affected by gDNA extraction methods, but only by soil type (Table 3). Fungal alpha diversity is not affected by soil type and only partly affected by gDNA extraction method (Table 3; Figure 4). This altogether indicates that our SDE method overall does not induce an experimental bias in extracting bacterial and fungal community data.

\section{CONCLUSION}

To conclude, we present a low-cost gDNA extraction method (\$0.36/sample, see S5, https://doi.org/10.5281/zenodo.4060156 for detailed breakdown) that effectively extracts soil samples and delivers good quality and quantities of gDNA suitable for microbiome studies. We show that the SDE method does not introduce a library preparation and therefore a sequencing bias. We also present a low-cost custom and fully Illumina-compatible bacterial and fungal amplicon library construction protocol that can multiplex up to 2304 samples to one pool ( $\$ 2.5 /$ library). Our method, therefore, enables researchers to sequence their projects on any available Illumina platform without the need to purchase full lanes/flow cells. Overall, the two presented methods will enable microbiome projects to be performed at any desired scale at an affordable price for a broad audience of microbiome enthusiasts.

\section{ETHICS STATEMENT}

None required.

\section{ACKNOWLEDGMENTS}

We thank Sarah Walkington at the Natural History Museum, London, for assistance with sequencing. Financial support was provided by 
a John Innes Foundation Rotation PhD Studentship to A.B.G and Natural History Museum support to M.D.C. Work in the Malone lab is funded by Biotechnology and Biological Sciences Research Council (BBSRC) Institute Strategic Programme grant BBS/E/J/000PR9797 (Plant Health) to the John Innes Centre.

\section{CONFLICT OF INTEREST}

None declared.

\section{AUTHOR CONTRIBUTION}

Anita Bollmann-Giolai: Conceptualization (supporting); Formal analysis (lead); Investigation (lead); Methodology (supporting); Writing-original draft (lead); Writing-review \& editing (supporting). Michael Giolai: Data curation (supporting); Formal analysis (supporting); Investigation (supporting); Methodology (supporting). Darren Heavens: Investigation (supporting); Methodology (supporting); Resources (supporting). lain Macaulay: Investigation (supporting); Methodology (supporting); Project administration (supporting); Resources (supporting). Jacob Malone: Conceptualization (supporting); Formal analysis (supporting); Funding acquisition (supporting); Methodology (supporting); Resources (lead); Supervision (lead). Matthew Derek Clark: Conceptualization (lead); Methodology (lead); Supervision (supporting); Writing-review \& editing (lead).

\section{DATA AVAILABILITY STATEMENT}

The sequence datasets generated during the current study are available in the European Nucleotide Archive (ENA) repository: https:// www.ebi.ac.uk/ena/browser/view/PRJEB37921. Supporting Information files are available in Zenodo: https://doi.org/10.5281/ zenodo.4060156.

\section{ORCID}

Anita Bollmann-Giolai (D) https://orcid.org/0000-0002-4398-7509

Michael Giolai (D) https://orcid.org/0000-0003-4166-8202

Darren Heavens (D) https://orcid.org/0000-0001-5418-7868

lain Macaulay (D) https://orcid.org/0000-0002-6761-757X

Jacob Malone (D) https://orcid.org/0000-0003-1959-6820

Matthew D. Clark (D) https://orcid.org/0000-0002-8049-5423

\section{REFERENCES}

Abdel-Latif, A., \& Osman, G. (2017). Comparison of three genomic DNA extraction methods to obtain high DNA quality from maize. Plant Methods, 13, 1. https://doi.org/10.1186/s13007-016-0152-4

Augusto, L., Ranger, J., Binkley, D., \& Rothe, A. (2002). Impact of several common tree species of European temperate forests on soil fertility. Annals of Forest Science, 59, 233-253. https://doi.org/10.1051/ forest:2002020

Bahram, M., Hildebrand, F., Forslund, S. K., Anderson, J. L., Soudzilovskaia, N. A., Bodegom, P. M., Anslan, S., Coelho, L. P., Harend, H., \& Huerta-Cepas, J. (2018). Structure and function of the global topsoil microbiome. Nature, 560, 233-237. https://doi.org/10.1038/ s41586-018-0386-6

Bartoli, C., Frachon, L., Barret, M., Rigal, M., Huard-Chauveau, C., Mayjonade, B., Zanchetta, C., Bouchez, O., Roby, D., Carrère, S., $\&$ Roux, F. (2018). In situ relationships between microbiota and potential pathobiota in Arabidopsis thaliana. The ISME Journal, 12(8), 2024-2038. https://doi.org/10.1038/s41396-018-0152-7

Brolaski, M. N., Venugopal, R. J., \& Stolow, D. (2008). United States Patent: 7459548 - Kits and processes for removing contaminants from nucleic acids in environmental and biological samples. Retrieved from http://patft.uspto.gov/netacgi/nph-Parser?Sect1=PTO1\&Sect2 $=$ HITOFF\& $d=P A L L \& p=1 \& u=\% 2 F$ netahtml\%2FPTO\%2Fsrchnum. $h t m \& r=1 \& f=G \& l=50 \& s 1=7,459,548 . P N . \& O S=P N / 7,459,548 \& R S$ $=\mathrm{PN} / 7,459,548$

Bürgmann, H., Pesaro, M., Widmer, F., \& Zeyer, J. (2001). A strategy for optimizing quality and quantity of DNA extracted from soil. Journal of Microbiological Methods, 45, 7-20. https://doi.org/10.1016/ S0167-7012(01)00213-5

Callahan, B. J., Sankaran, K., Fukuyama, J. A., McMurdie, P. J., \& Holmes, S. P. (2016). Bioconductor workflow for microbiome data analysis: From raw reads to community analyses. F1000Research, 5, 1492. https://doi.org/10.12688/f1000research.8986.1

Devi, S. G., Fathima, A. A., Radha, S., Arunraj, R., Curtis, W. R., \& Ramya, M. (2015). A rapid and economical method for efficient DNA extraction from diverse soils suitable for metagenomic applications. PLoS One, 10, e0132441. https://doi.org/10.1371/journ al.pone. 0132441

Dixon, P. (2003). VEGAN, a package of R functions for community ecology. Journal of Vegetation Science, 14, 927-930. https://doi. org/10.1111/j.1654-1103.2003.tb02228.x

Dong, D., Yan, A., Liu, H., Zhang, X., \& Xu, Y. (2006). Removal of humic substances from soil DNA using aluminium sulfate. Journal of Microbiological Methods, 66, 217-222. https://doi.org/10.1016/j. mimet.2005.11.010

Fatima, F., Pathak, N., \& Rastogi, V. S. (2014). An improved method for soil DNA extraction to study the microbial assortment within Rhizospheric Region. Molecular Biology International, 2014, 1-6. https://doi.org/10.1155/2014/518960

Fisher, S., Barry, A., Abreu, J., Minie, B., Nolan, J., Delorey, T. M., Young, G., Fennell, T. J., Allen, A., Ambrogio, L., Berlin, A. M., Blumenstiel, B., Cibulskis, K., Friedrich, D., Johnson, R., Juhn, F., Reilly, B., Shammas, R., Stalker, J., ... Nusbaum, C. (2011). A scalable, fully automated process for construction of sequence-ready human exome targeted capture libraries. Genome Biology, 12, R1. https:// doi.org/10.1186/gb-2011-12-1-r1

Ginestet, C. (2011). ggplot2: Elegant Graphics for Data Analysis. J R Stat Soc Ser A Stat Soc., 174, 245-246.

Giolai, M., Verweij, W., Lister, A., Heavens, D., Macaulay, I., \& Clark, M. D. (2019). Spatially resolved transcriptomics reveals plant host responses to pathogens. Plant Methods, 15, 114. https://doi. org/10.1186/s13007-019-0498-5

Hamedi, J., Danaiefar, M., \& Moghimi, H. (2016). Rapid and efficient method for environmental DNA extraction and purification from soil. Journal of Microbiology, Biotechnology and Food Sciences, 05, 530-533. https://doi.org/10.15414/jmbfs.2016.5.6.530-533

Kelly, B. J., Gross, R., Bittinger, K., Sherrill-Mix, S., Lewis, J. D., Collman, R. G., Bushman, F. D., \& Li, H. (2015). Power and sample-size estimation for microbiome studies using pairwise distances and PERMANOVA. Bioinformatics, 31, 2461-2468. https://doi.org/10.1093/bioinforma tics/btv183

Lakay, F. M., Botha, A., \& Prior, B. A. (2007). Comparative analysis of environmental DNA extraction and purification methods from different humic acid-rich soils. Journal of Applied Microbiology, 102, 265-273. https://doi.org/10.1111/j.1365-2672.2006.03052.x

Lundberg, D. S., Yourstone, S., Mieczkowski, P., Jones, C. D., \& Dangl, J. L. (2013). Practical innovations for high-throughput amplicon sequencing. Nature Methods, 10, 999-1002. https://doi.org/10.1038/ nmeth.2634

Mandalakis, M., Panikov, N. S., Polymenakou, P. N., Sizova, M. V., \& Stamatakis, A. (2018). A simple cleanup method for the removal of 
humic substances from soil protein extracts using aluminum coagulation. Environmental Science and Pollution Research, 25, 2384523856. https://doi.org/10.1007/s11356-018-2434-z

Marotz, C., Amir, A., Humphrey, G., Gaffney, J., Gogul, G., \& Knight, R. (2017). DNA extraction for streamlined metagenomics of diverse environmental samples. BioTechniques, 62, 290-293. https://doi. org/10.2144/000114559

Martin, M. (2011). Cutadapt removes adapter sequences from high-throughput sequencing reads. EMBnet.journal, 17, 10-12. https://doi.org/10.14806/ej.17.1.200

McMurdie, P. J., \& Holmes, S. (2013). phyloseq: An R package for reproducible interactive analysis and graphics of microbiome census data. PLoS One, 8, e61217. https://doi.org/10.1371/journ al.pone.0061217

Miao, T., Gao, S., Jiang, S., Kan, G., Liu, P., Wu, X., An, Y., \& Yao, S. (2014). A method suitable for DNA extraction from humus-rich soil. Biotechnology Letters, 36, 2223-2228. https://doi.org/10.1007/ s10529-014-1591-5

Minich, J. J., Humphrey, G., Benitez, R. A. S., Sanders, J., Swafford, A., Allen, E. E., \& Knight, R. (2018). High-throughput miniaturized $16 S$ rRNA amplicon library preparation reduces costs while preserving microbiome integrity. mSystems, 3. https://doi.org/10.1128/mSyst ems.00166-18

Minich, J. J., Zhu, Q., Janssen, S., Hendrickson, R., Amir, A., Vetter, R., Hyde, J., Doty, M. M., Stillwell, K., Benardini, J., Kim, J. H., Allen, E. E., Venkateswaran, K., \& Knight, R. (2018). KatharoSeq enables high-throughput microbiome analysis from low-biomass samples. mSystems, 3, e00218-e317. https://doi.org/10.1128/mSyst ems.00218-17

Narayan, A., Jain, K., Shah, A. R., \& Madamwar, D. (2016). An efficient and cost-effective method for DNA extraction from athalassohaline soil using a newly formulated cell extraction buffer. 3 Biotech, 6. https://doi.org/10.1007/s13205-016-0383-0

Nilsson, R. H., Larsson, K.-H., Taylor, A. F. S., Bengtsson-Palme, J., Jeppesen, T. S., Schigel, D., Kennedy, P., Picard, K., Glöckner, F. O., Tedersoo, L., Saar, I., Kõljalg, U., \& Abarenkov, K. (2019). The UNITE database for molecular identification of fungi: Handling dark taxa and parallel taxonomic classifications. Nucleic Acids Research, 47, D259-D264. https://doi.org/10.1093/nar/gky1022

Oberacker, P., Stepper, P., Bond, D. M., Höhn, S., Focken, J., Meyer, V., Schelle, L., Sugrue, V. J., Jeunen, G. J., Moser, T., \& Hore, S. R. (2019). Bio-On-Magnetic-Beads (BOMB): Open platform for high-throughput nucleic acid extraction and manipulation. PLoS Biology, 17, e3000107. https://doi.org/10.1371/journal.pbio.3000107

Quast, C., Pruesse, E., Yilmaz, P., Gerken, J., Schweer, T., Yarza, P., Peplies, J., \& Glöckner, F. O. (2013). The SILVA ribosomal RNA gene database project: Improved data processing and web-based tools. Nucleic Acids Research, 41, D590-D596. https://doi.org/10.1093/ nar/gks1219

Robe, P., Nalin, R., Capellano, C., Vogel, T. M., \& Simonet, P. (2003). Extraction of DNA from soil. European Journal of Soil Biology, 39(4), 183-190. https://doi.org/10.1016/S1164-5563(03)00033-5

Rowan, B. A., Heavens, D., Feuerborn, T. R., Tock, A. J., Henderson, I. R., \& Weigel, D. (2019). An ultra high-density Arabidopsis thaliana crossover map that refines the influences of structural variation and epigenetic features. Genetics, 213, 771-787. https://doi. org/10.1534/genetics.119.302406
Sáenz, J. S., Roldan, F., Junca, H., \& Arbeli, Z. (2019). Effect of the extraction and purification of soil DNA and pooling of PCR amplification products on the description of bacterial and archaeal communities. Journal of Applied Microbiology, 126, 1454-1467. https://doi. org/10.1111/jam.14231

Schwarze, K., Buchanan, J., Fermont, J. M., et al. (2020). The complete costs of genome sequencing: a microcosting study in cancer and rare diseases from a single center in the United Kingdom. Genet Med., 22, 85-94. https://doi.org/10.1038/s41436-019-0618-7

Thiergart, T., Durán, P., Ellis, T., Vannier, N., Garrido-Oter, R., Kemen, E., Roux, F., Alonso-Blanco, C., Ågren, J., Schulze-Lefert, P., \& Hacquard, S. (2020). Root microbiota assembly and adaptive differentiation among European Arabidopsis populations. Nature Ecology \& Evolution, 4(1), 122-131. https://doi.org/10.1038/ s41559-019-1063-3

Tourlousse, D. M., Ohashi, A., \& Sekiguchi, Y. (2018). Sample tracking in microbiome community profiling assays using synthetic $16 \mathrm{~S}$ rRNA gene spike-in controls. Scientific Reports, 8, 1-9. https://doi. org/10.1038/s41598-018-27314-3

Verma, S. K., Singh, H., \& Sharma, P. C. (2017). An improved method suitable for isolation of high-quality metagenomic DNA from diverse soils. 3 Biotech, 7(3). https://doi.org/10.1007/s13205-017-0847-x

Walters, W., Hyde, E. R., Berg-Lyons, D., Ackermann, G., Humphrey, G., Parada, A., Gilbert, J. A., Jansson, J. K., Caporaso, J. G., Fuhrman, J. A., Apprill, A., \& Knight, R. (2016). Improved bacterial 16 S rRNA gene (V4 and $\mathrm{V} 4-5$ ) and fungal internal transcribed spacer marker gene primers for microbial community surveys. mSystems., 1, e00009-e00015. https://doi.org/10.1128/mSystems.00009-15

Walters, W. A., Jin, Z., Youngblut, N., Wallace, J. G., Sutter, J., Zhang, W., González-Peña, A., Peiffer, J., Koren, O., Shi, Q., \& Knight, R. (2018). Large-scale replicated field study of maize rhizosphere identifies heritable microbes. Proceedings of the National Academy of Sciences of the United States of America, 115, 7368-7373. https:// doi.org/10.1073/pnas.1800918115

Yeates, C., Gillings, M. R., Davison, A. D., Altavilla, N., \& Veal, D. A. (1998). Methods for microbial DNA extraction from soil for PCR amplification. Biological Procedures Online, 1(1), 40-47. https://doi. org/10.1251/bpo6

Yu, Z., \& Mohn, W. W. (1999). Killing two birds with one stone: Simultaneous extraction of DNA and RNA from activated sludge biomass. Canadian Journal of Microbiology, 45, 269-272. https://doi. org/10.1139/w98-211

Zhou, J., Bruns, M. A., \& Tiedje, J. M. (1996). DNA recovery from soils of diverse composition. Applied and Environment Microbiology, 62, 316-322. https://doi.org/10.1128/AEM.62.2.316-322.1996

Zou, Y., Mason, M. G., Wang, Y., Wee, E., Turni, C., Blackall, P. J., Trau, M., \& Botella, J. R. (2017). Nucleic acid purification from plants, animals and microbes in under 30 seconds. PLoS Biology, 15, e2003916. https://doi.org/10.1371/journal.pbio.2003916

How to cite this article: Bollmann-Giolai A, Giolai M, Heavens D, Macaulay I, Malone J, Clark MD. A low-cost pipeline for soil microbiome profiling. MicrobiologyOpen. 2020;00:e1133. https://doi.org/10.1002/mbo3.1133 


\section{APPENDIX A}

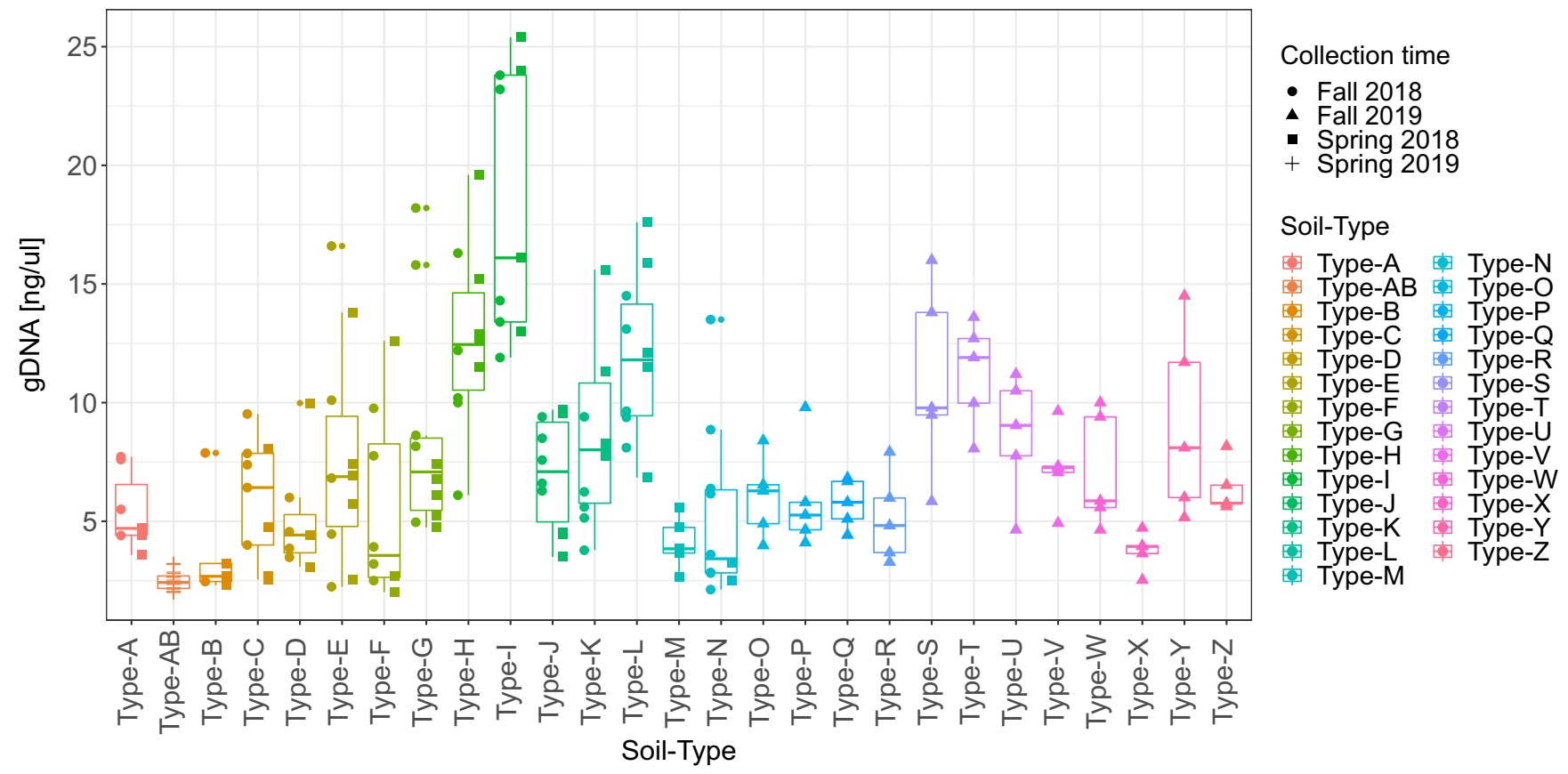

Figure A1 Concentration of gDNA of plate extracted gDNA from 27 different soil types of 196 samples collected throughout four seasons (spring 2018, fall 2018, spring 2019, fall 2019). gDNA, genomic DNA 


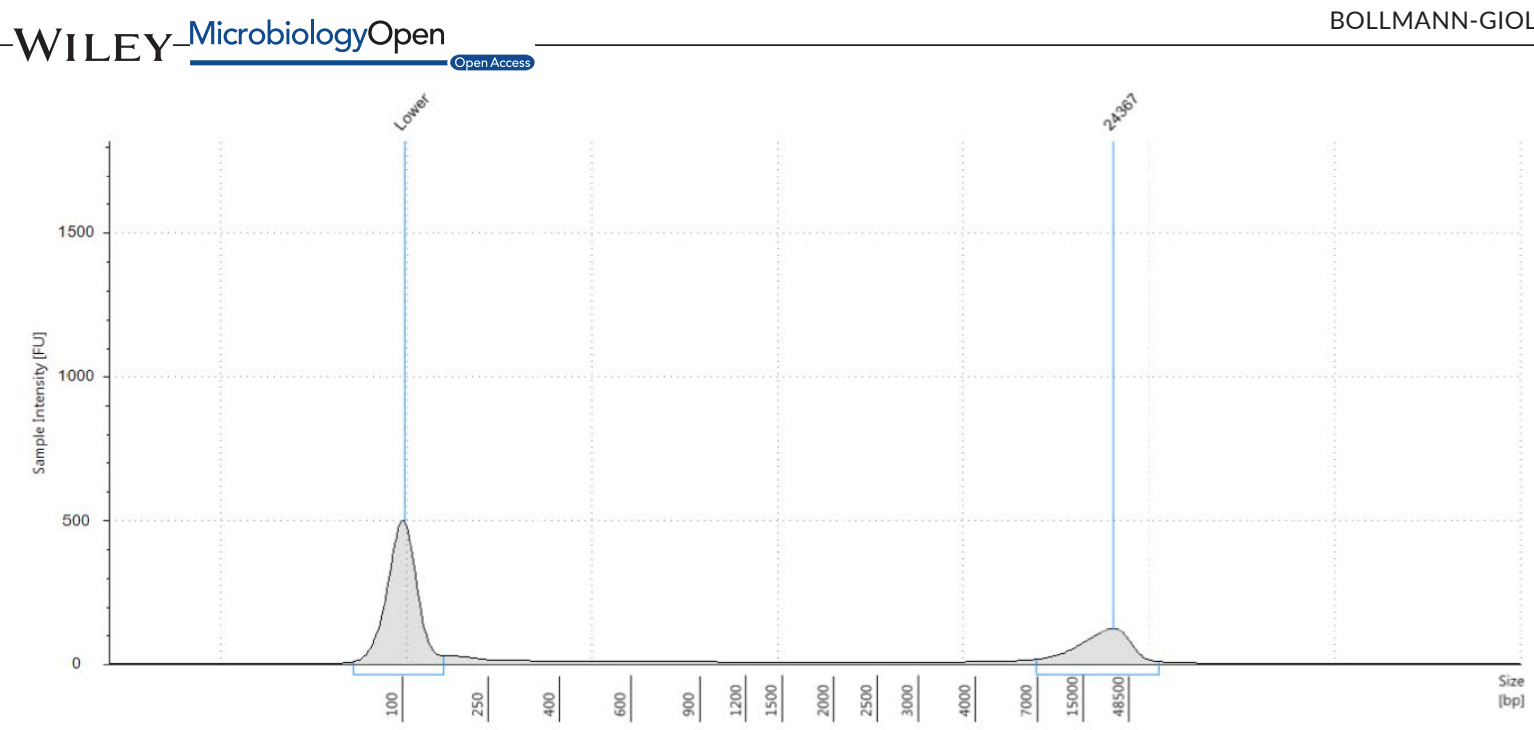

(a) Tapestation gDNA trace MoBio PowerSoil ${ }^{\circledR}$ for representative Cer (John Innes cereal compost mix) sample.

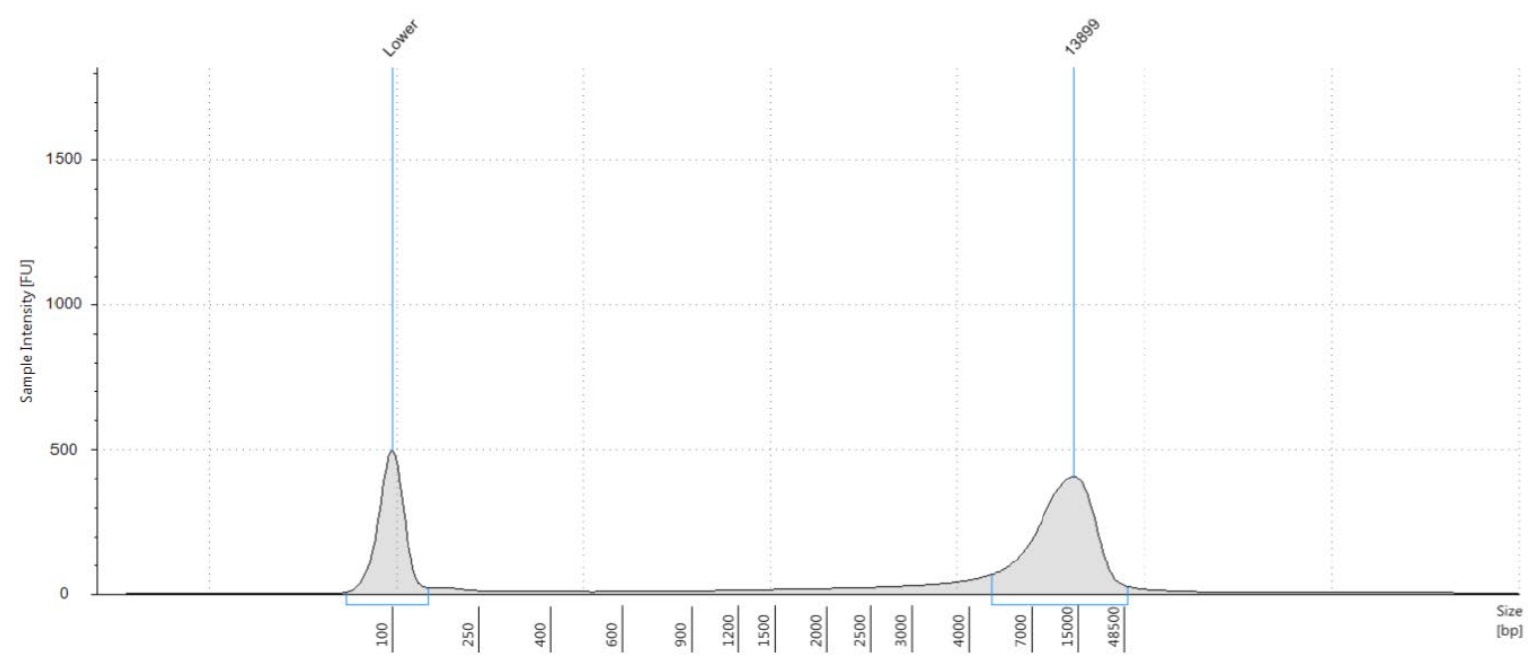

(b) Tapestation gDNA trace MoBio PowerSoil ${ }^{\circledR}$ for representative MiF (mixed forest) sample.

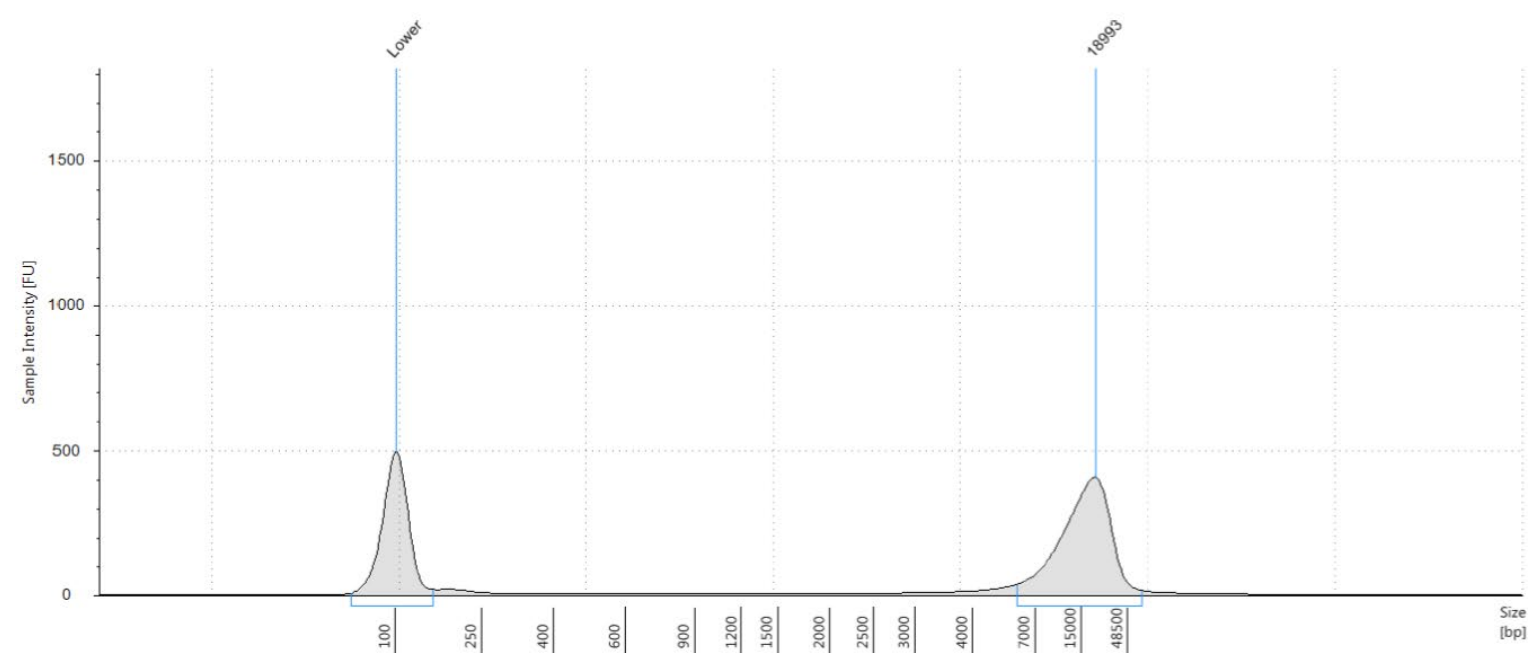

(c) Tapestation gDNA trace MoBio PowerSoil ${ }^{\circledR}$ for representative BrF (broad leafed forest) sample. 


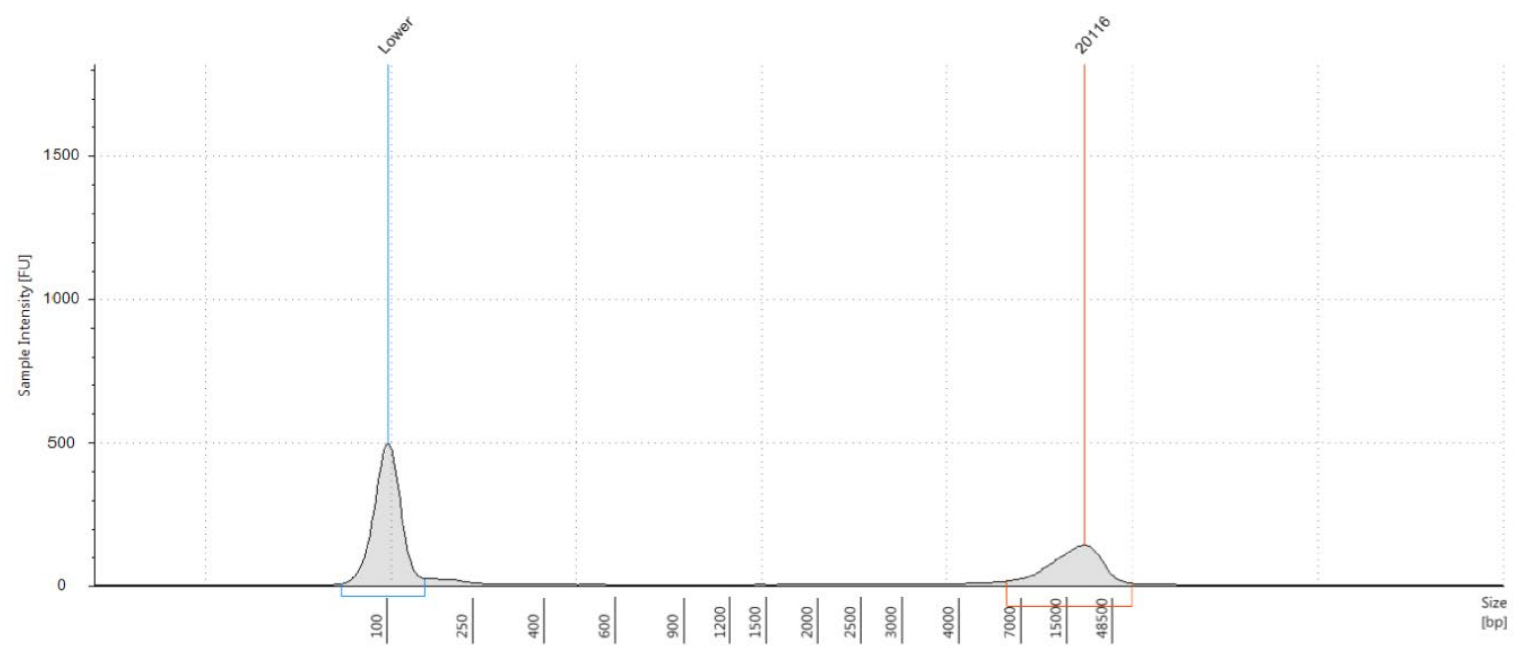

(d) Tapestation gDNA trace MoBio PowerSoil ${ }^{\circledR}$ for representative CoF (coniferous forest) sample.

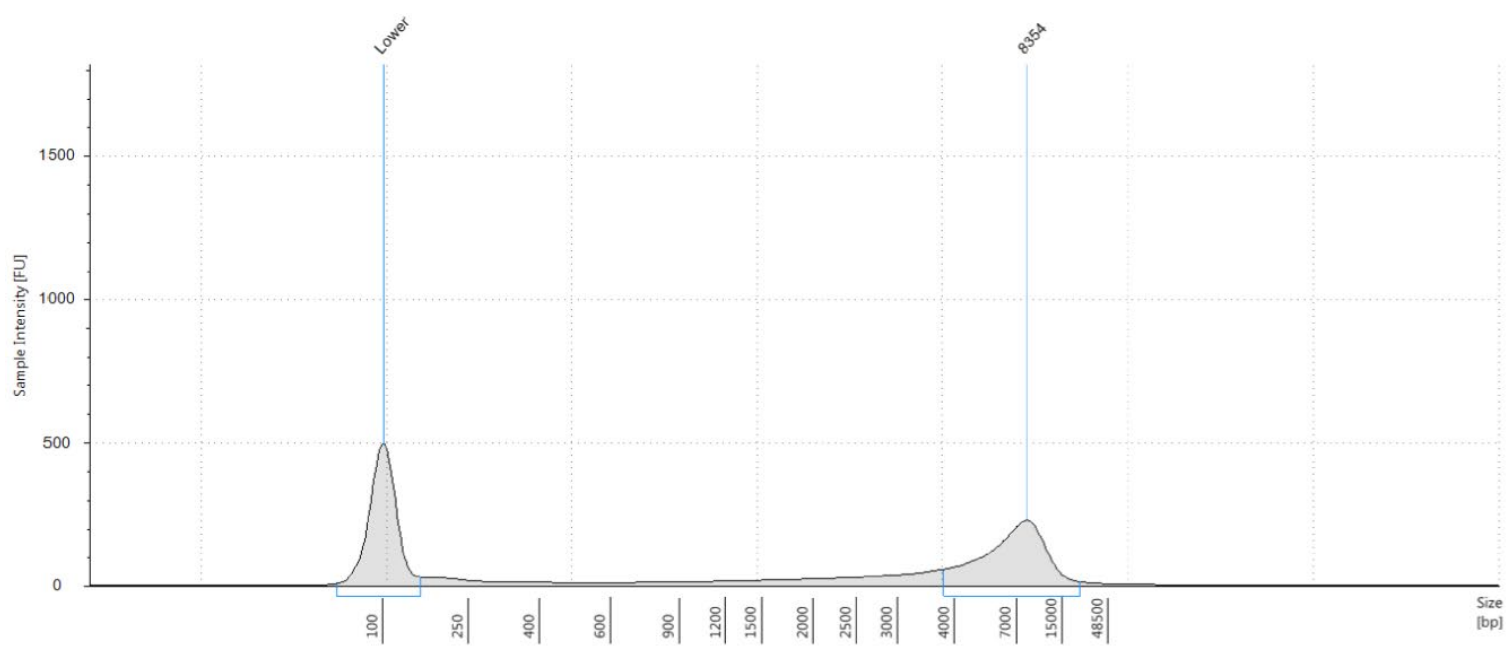

(e) Tapestation gDNA trace MP Biomedicals ${ }^{\mathrm{TM}}$ FastDNA $^{\mathrm{TM}}$ SPIN kit for representative Cer (John Innes cereal compost mix) sample.

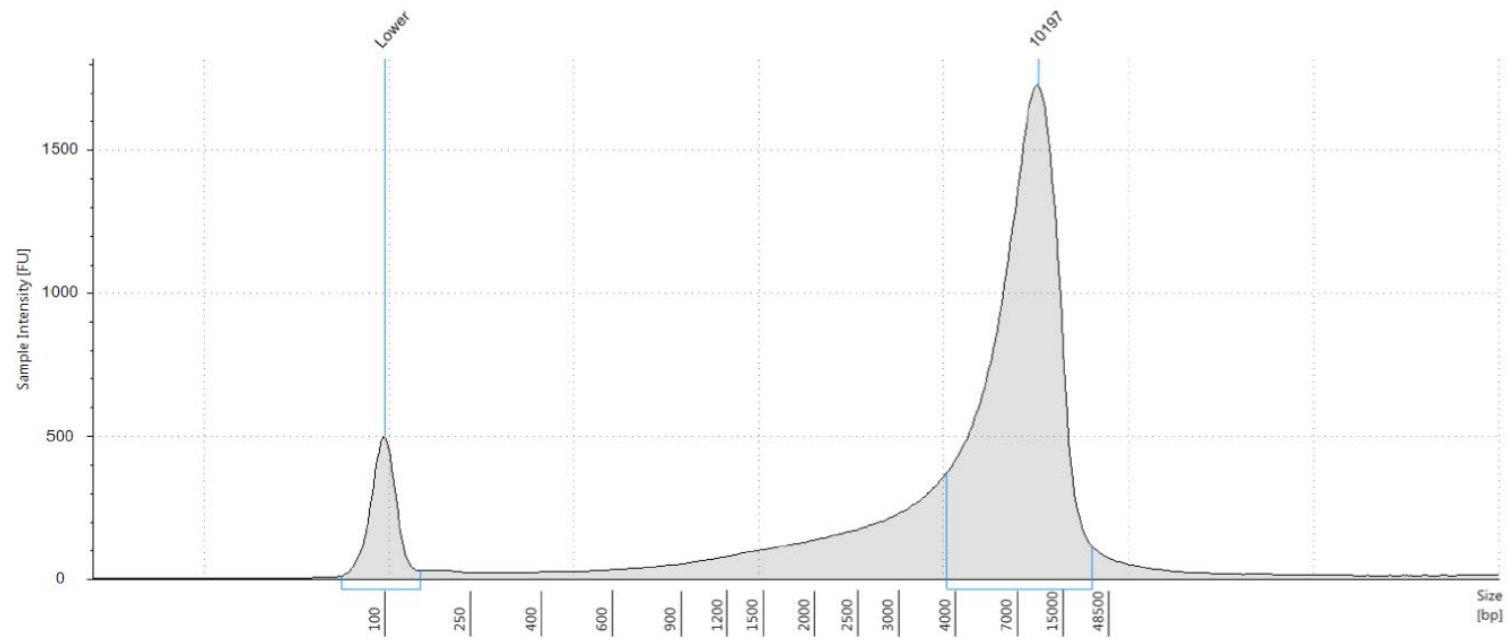

(f) Tapestation gDNA trace MP Biomedicals ${ }^{\mathrm{TM}}$ FastDNA $^{\mathrm{TM}}$ SPIN kit for representative MiF (mixed forest) sample.

Figure A2 (Continued) 


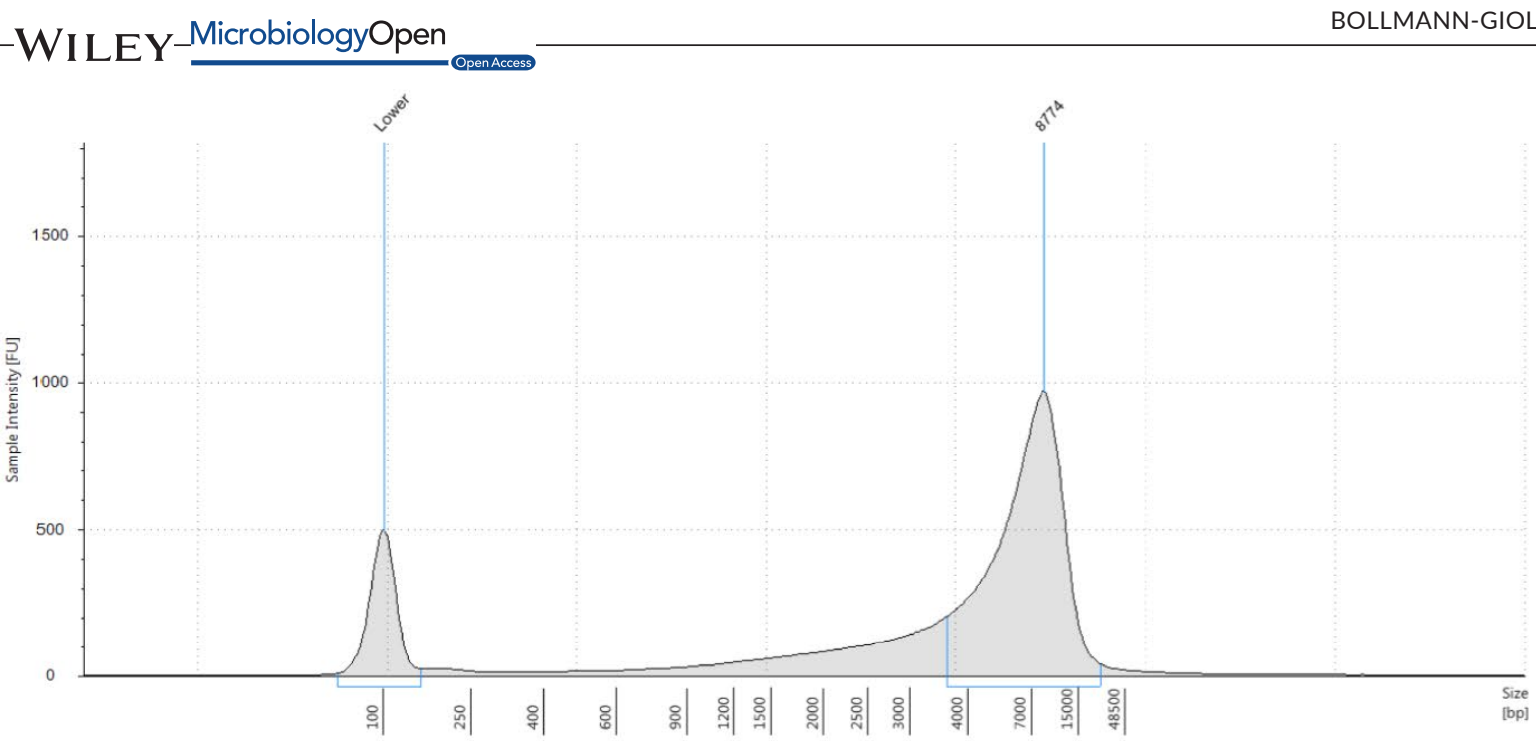

(g) Tapestation gDNA trace MP Biomedicals ${ }^{\mathrm{TM}}$ FastDNA $^{\mathrm{TM}}$ SPIN kit for representative BrF (broad leafed forest) sample.

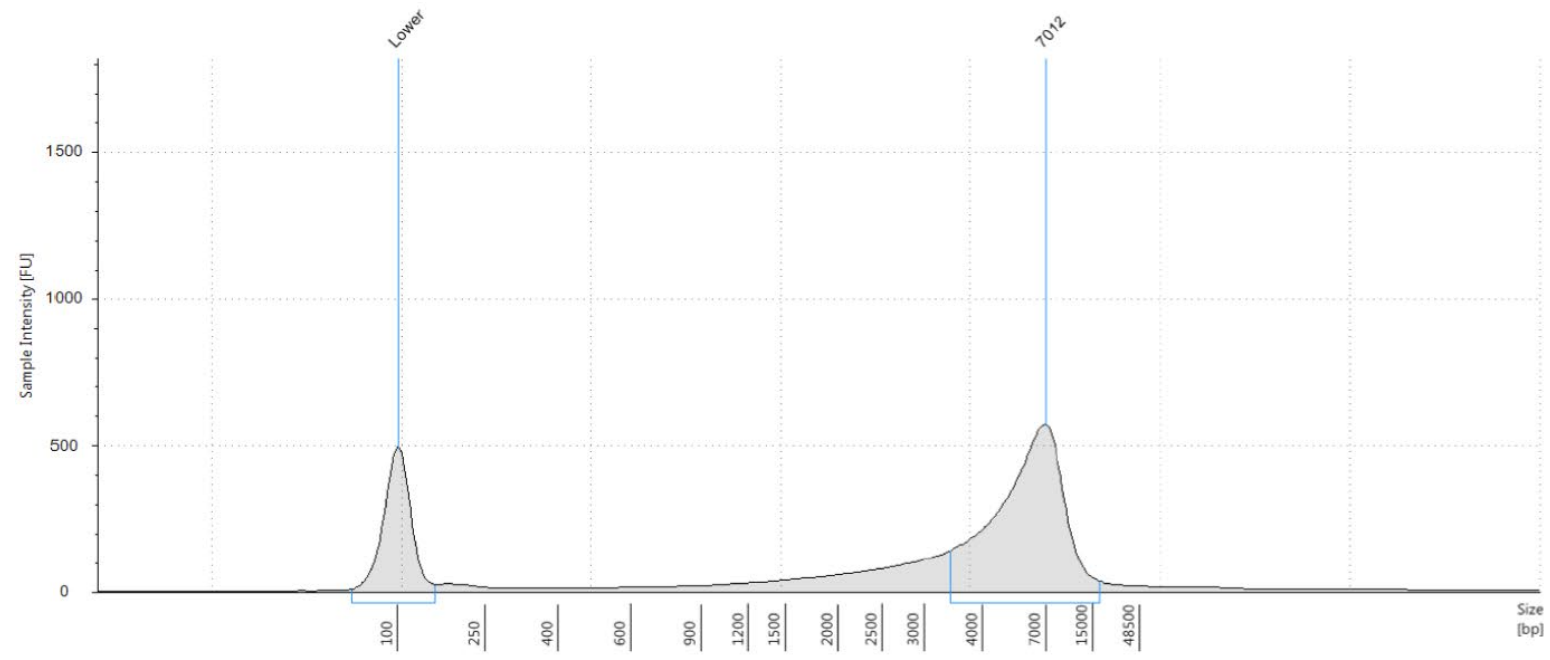

(h) Tapestation gDNA trace MP Biomedicals ${ }^{\mathrm{TM}}$ FastDNA $^{\mathrm{TM}}$ SPIN kit for representative CoF (coniferour forest) sample.

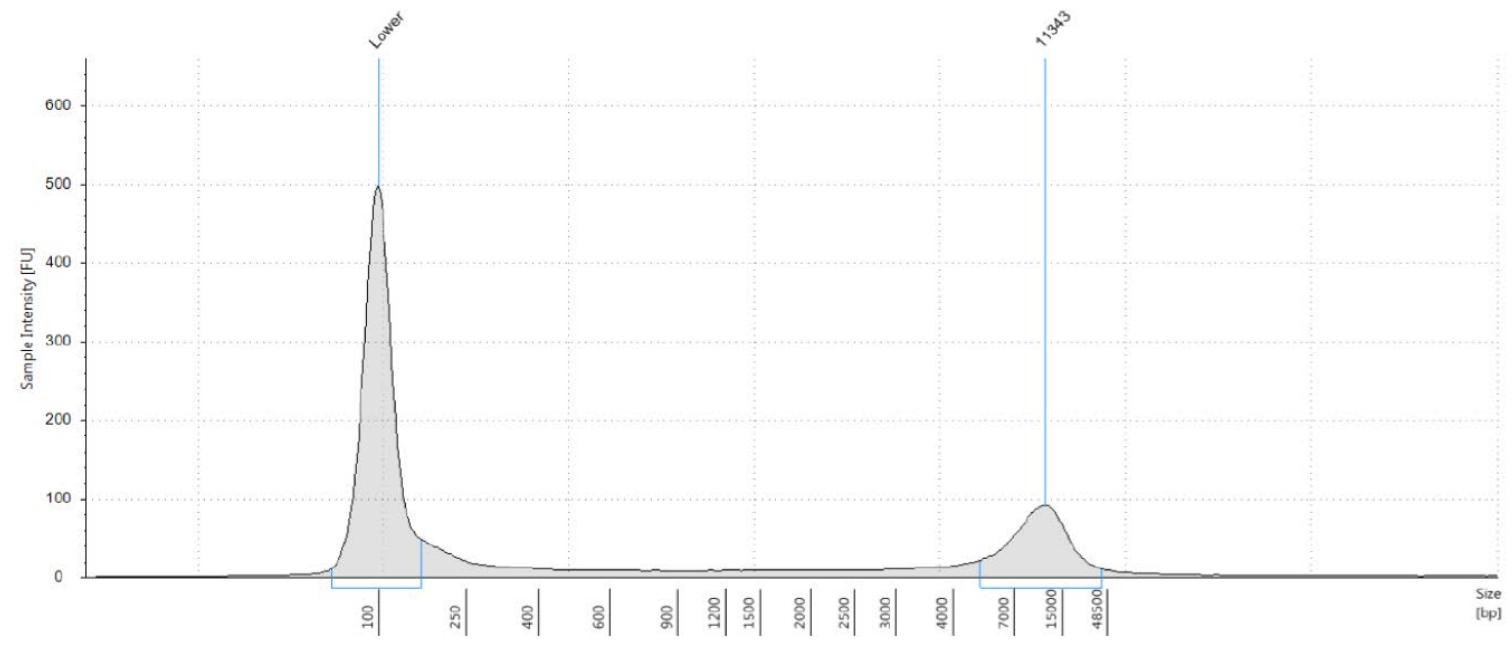

(i) Tapestation gDNA trace SDE method for representative CeR (John Innes cereal compost mix) sample.

Figure A2 (Continued) 


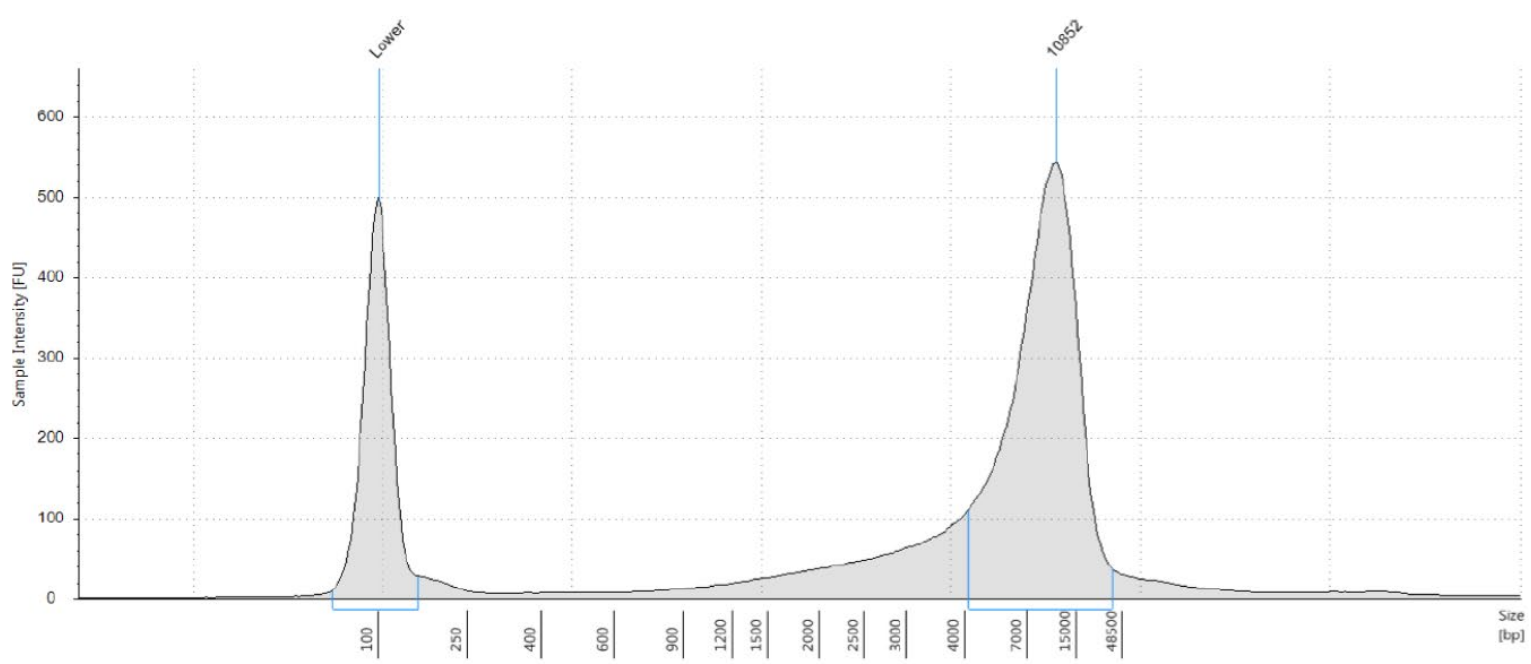

(j) Tapestation gDNA trace SDE method for representative MiF (mixed forest) sample.

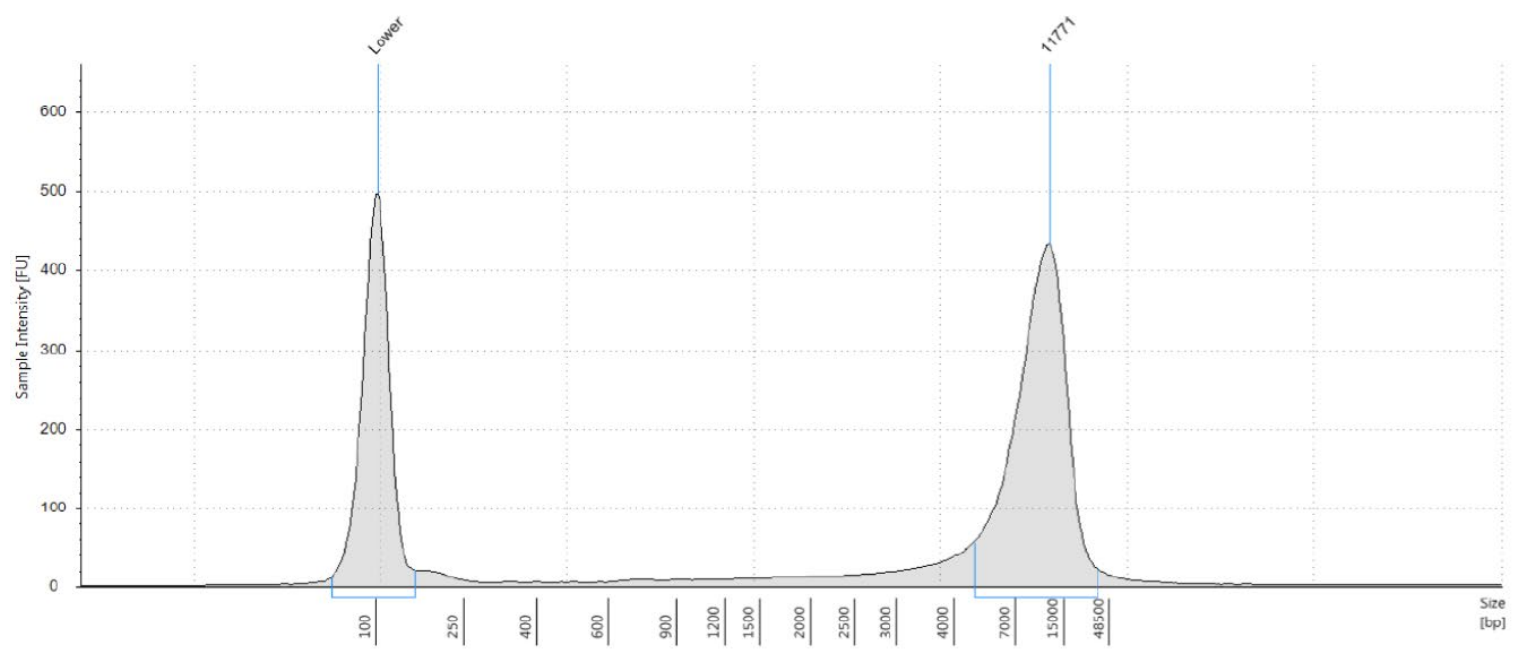

(k) Tapestation gDNA trace SDE method for representative BrF (broad leafed forest) sample.

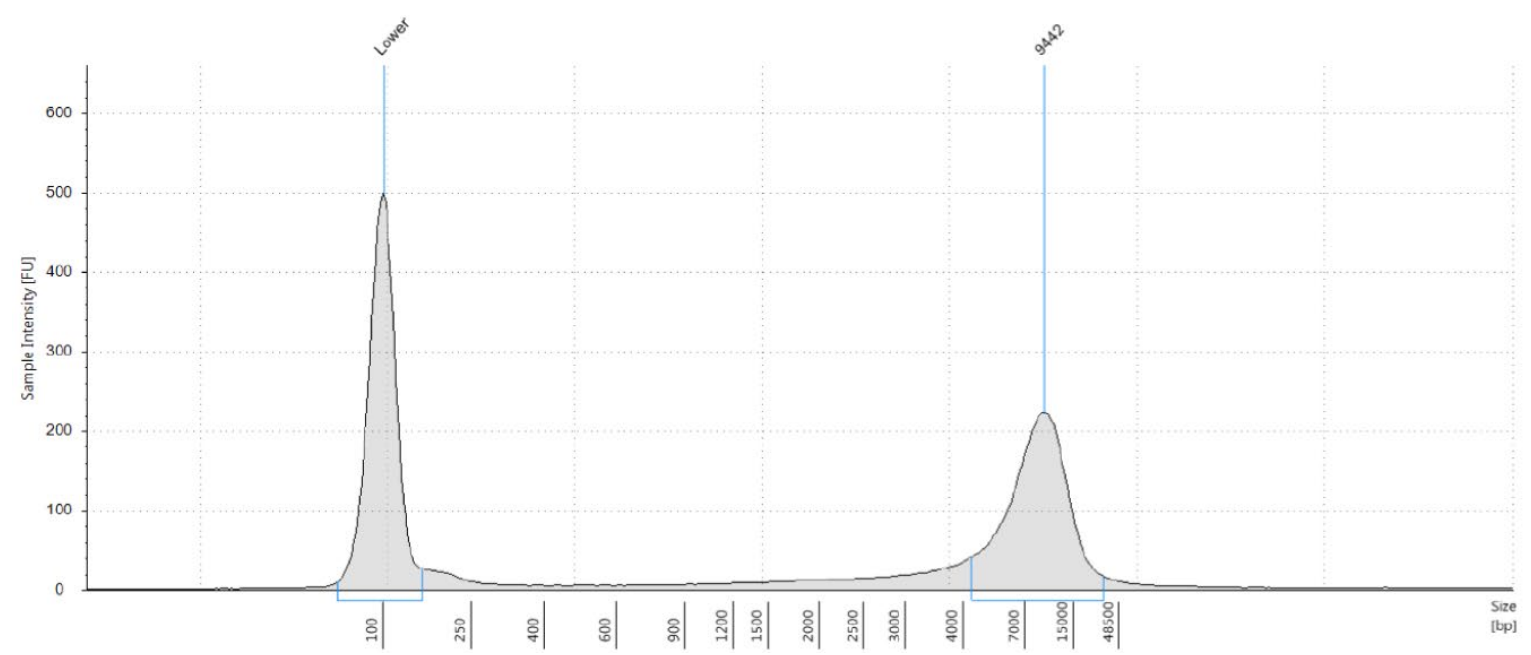

(I) Tapestation gDNA trace SDE method for representative CoF (coniferous forest) sample.

Figure A2 Genomic TapeStation traces of representative MoBio PowerSoil ${ }^{\circledR}$, MP Biomedicals ${ }^{\mathrm{TM}}$ FastDNA $^{\mathrm{TM}}$ SPIN and SDE gDNA extractions. gDNA, genomic DNA; SDE, soil gDNA extraction method 
(a) $16 \mathrm{~S}$

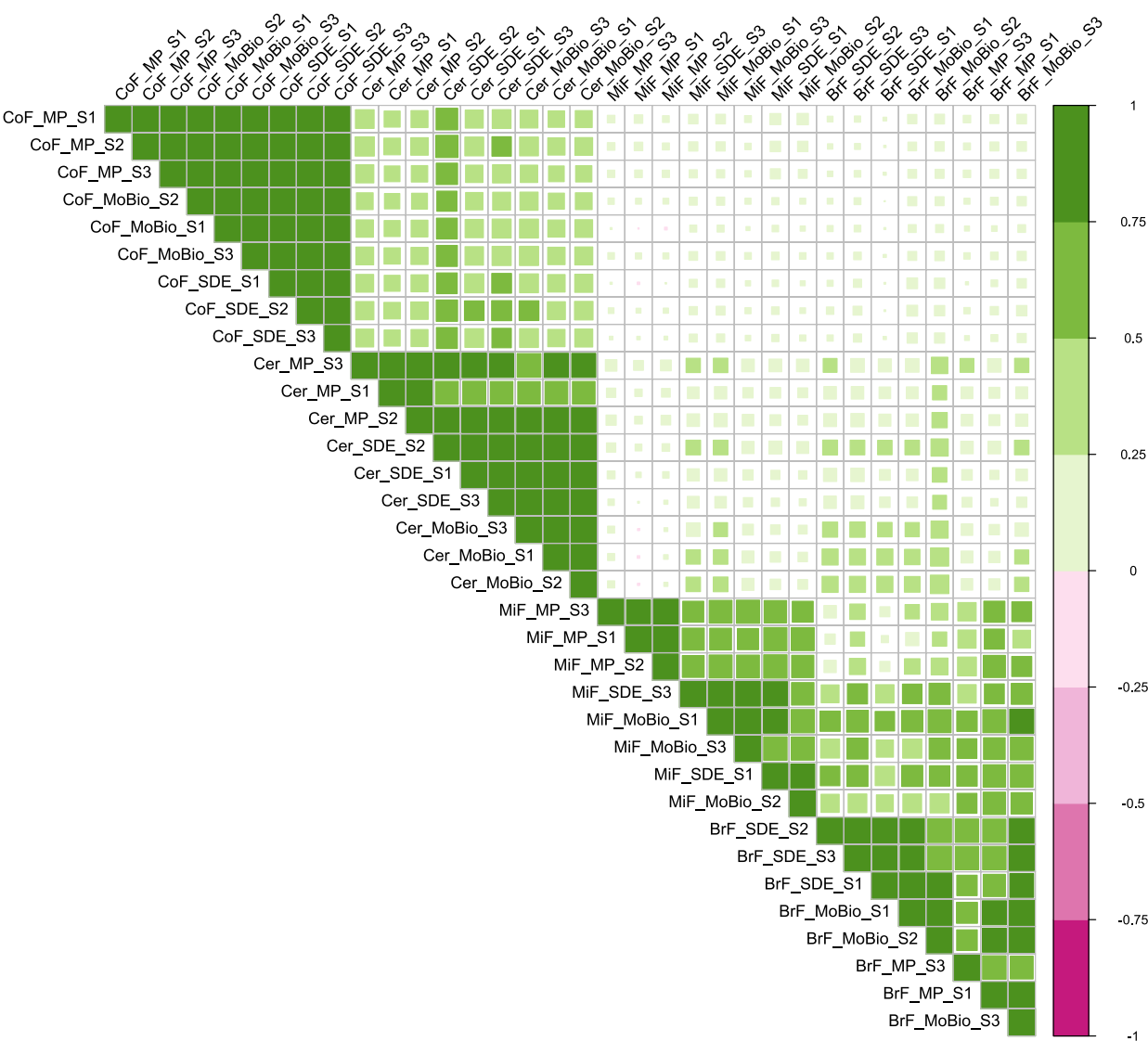

(b) ITS

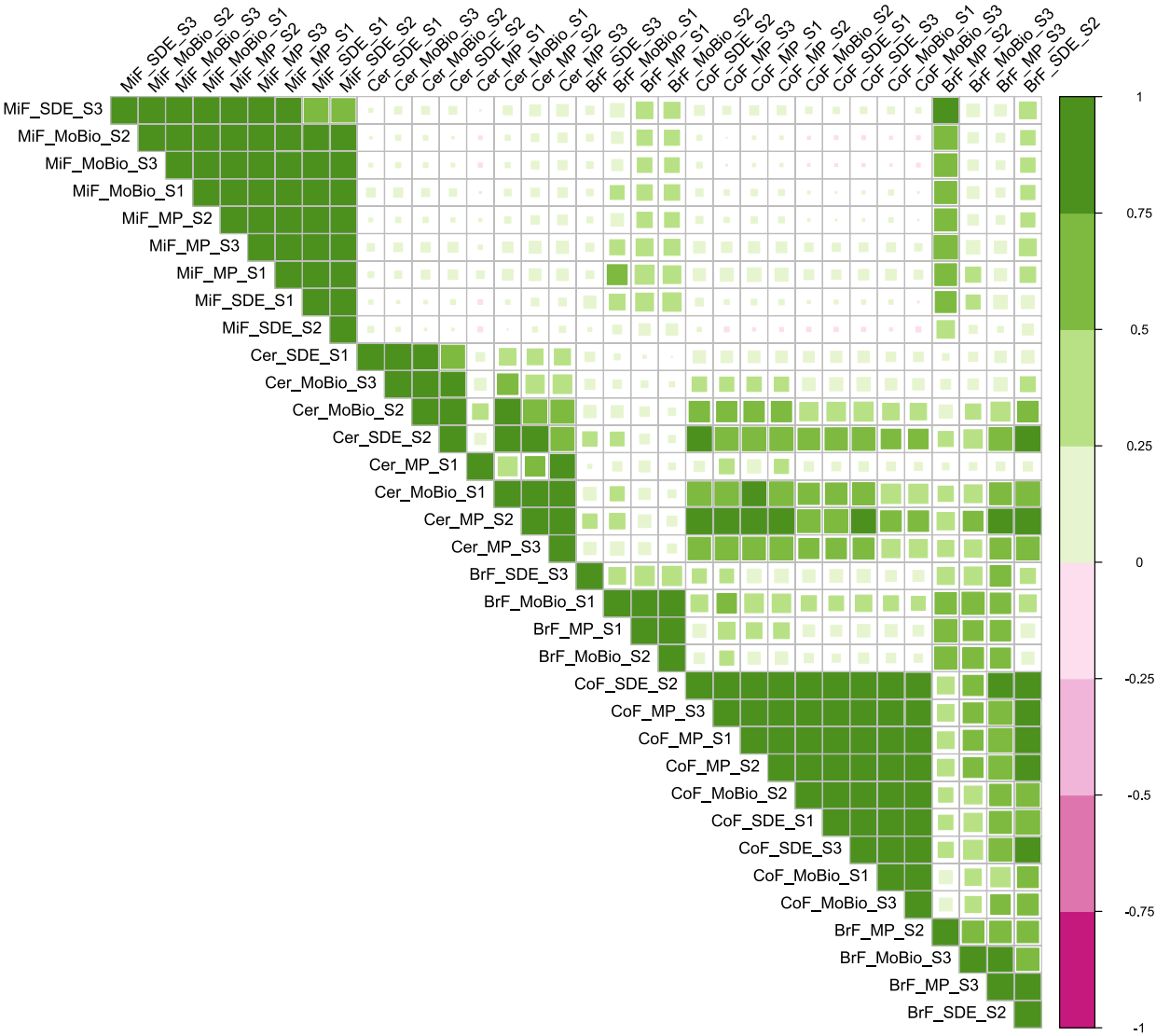

Figure A3 Corrplot of technical replicates of MP Biomedicals ${ }^{\mathrm{TM}}$ FastDNA ${ }^{\mathrm{TM}}$ SPIN, MoBio PowerSoil ${ }^{\circledR}$ and SDE method. Order level. SDE, soil gDNA extraction method 
(a)

16S: MP/SDE

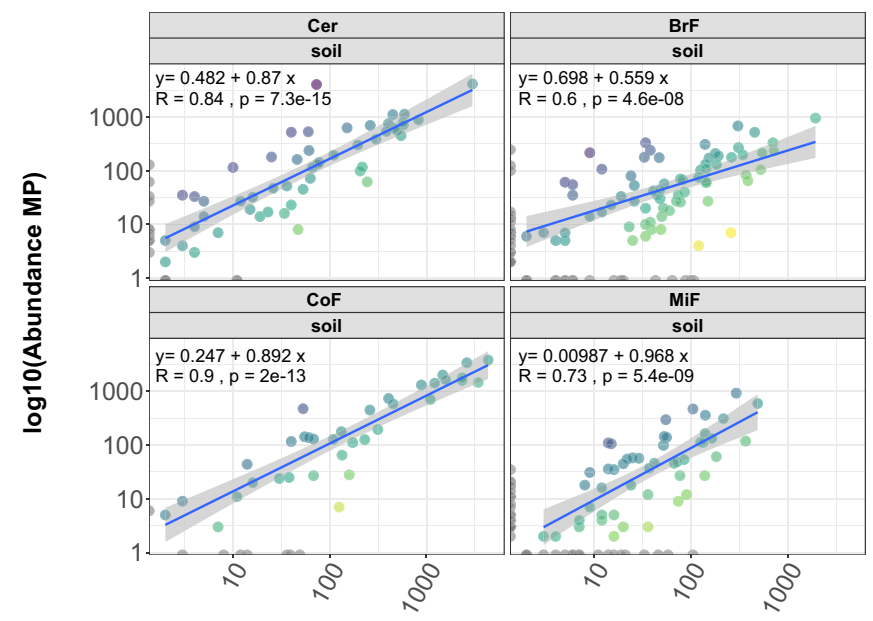

log10(Abundance SDE)

$\log 2(\mathrm{SDE} / \mathrm{MP})$

5.0
2.5
0.0
-2.5
-5.0

(c) ITS: MP/SDE

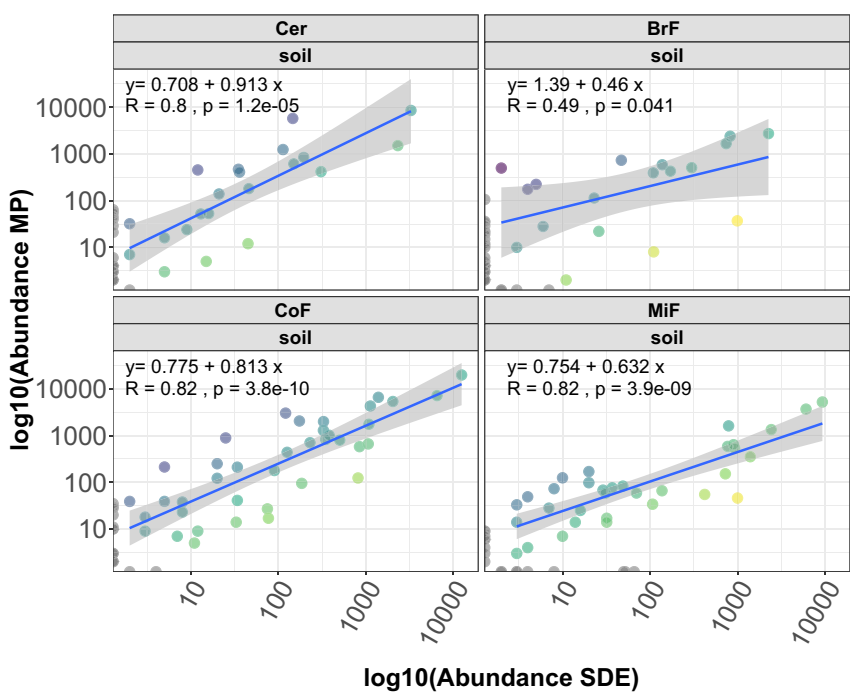

$\log 2(S D E / M P)$

2.5

0.0
-2.5

$-5.0$

$-7.5$ (b)

16S: MP/MoBio

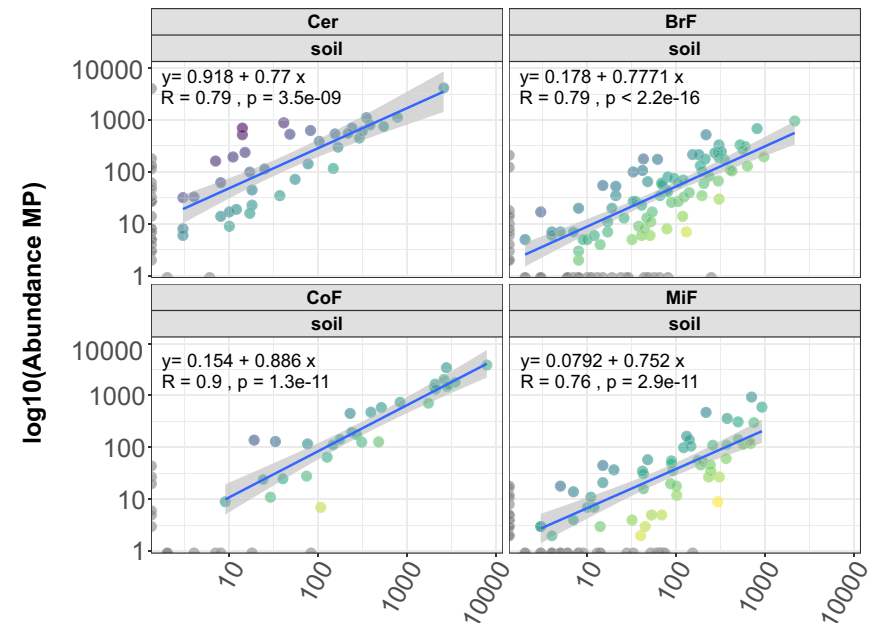

$\log 10$ (Abundance MoBio)

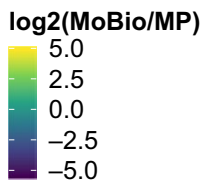

(d) ITS: MP/MoBio

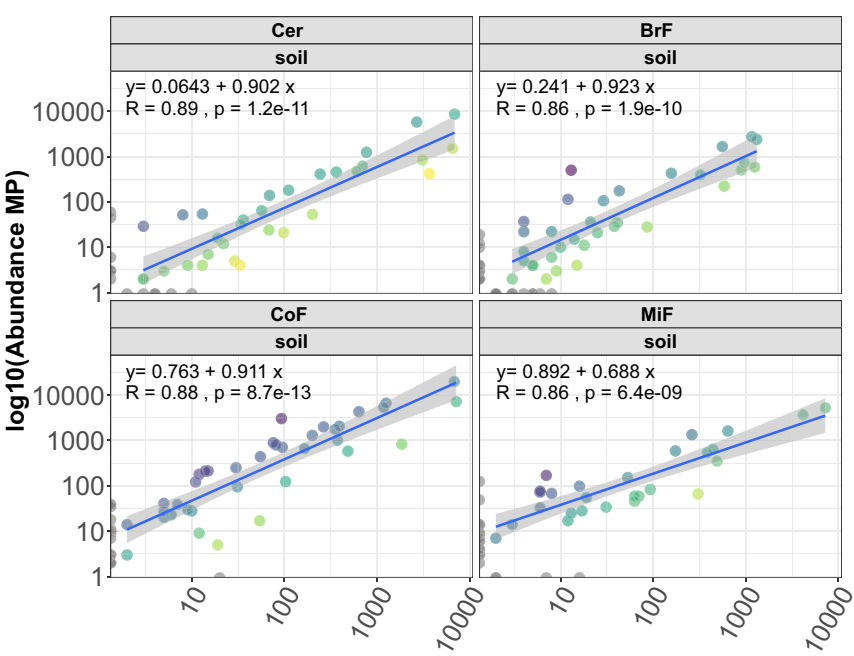

log10(Abundance MoBio)

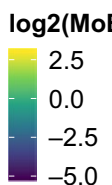

Figure A4 Correlation analysis SDE method versus MP Biomedicals ${ }^{\mathrm{TM}}$ FastDNA $^{\mathrm{TM}}$ SPIN \& MP Biomedicals ${ }^{\mathrm{TM}}$ FastDNA ${ }^{\mathrm{TM}}$ SPIN versus MoBio PowerSoil $(16 \mathrm{~S} / \mathrm{ITS})$. SDE, soil gDNA extraction method 